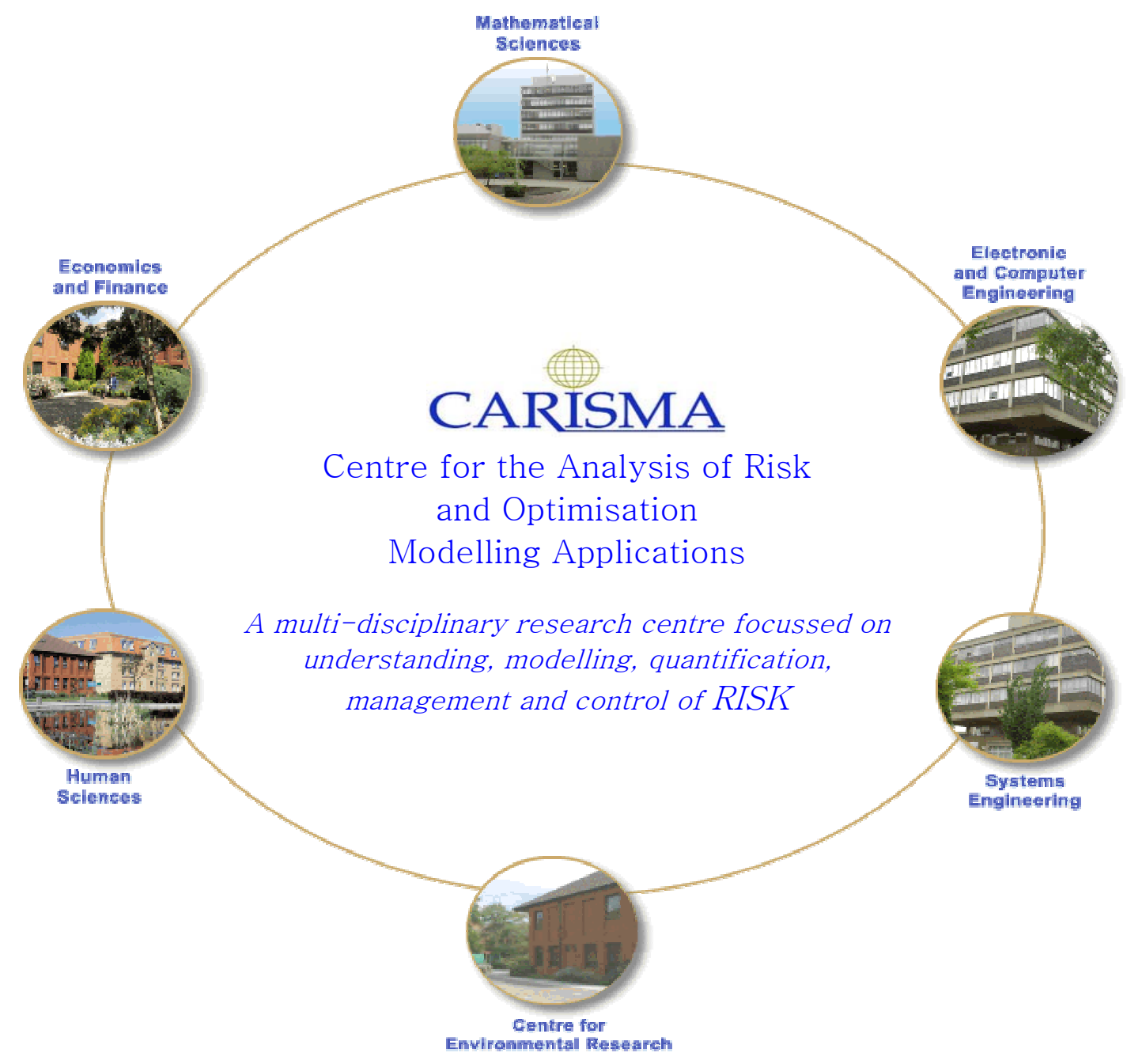

TECHNICAL REPORT

\begin{tabular}{||l||}
\hline CTR/09/03 November \\
Extending algebraic modelling languages for \\
Stochastic Programming \\
\\
Patrick Valente, Gautam Mitra and M Sadki \\
Brunel University, Uxbridge, Middlesex \\
R Fourer \\
Northwester University, Evanston, IL \\
\hline
\end{tabular}




\title{
Extending algebraic modelling languages for Stochastic Programming
}

\author{
P. Valente, G. Mitra, M. Sadki
}

Brunel University, Uxbridge, Middlesex, UK

\author{
R. Fourer
}

Northwestern University, Evanston, IL 


\begin{abstract}
The algebraic modelling languages (AML) have gained wide acceptance and use in Mathematical Programming by researchers and practitioners. At a basic level, stochastic programming models can be defined using these languages by constructing their deterministic equivalent. Unfortunately, this leads to very large model data instances. We propose a direct approach in which the random values of the model coefficients and the stage structure of the decision variables and constraints are "overlaid" on the underlying deterministic (core) model of the SP problems. This leads not only to a natural definition of the SP model: the resulting generated instance is also a compact representation of the otherwise large problem data. The proposed constructs enable the formulation of two stage and multistage scenario based recourse problems, as well as chance constrained problems. This design is presented as a stochastic extension of the AMPL language which we call SAMPL.
\end{abstract}




\section{Table of Contents}

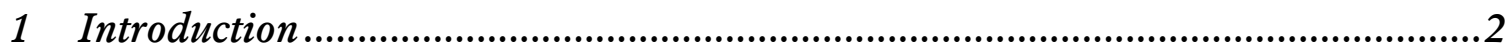

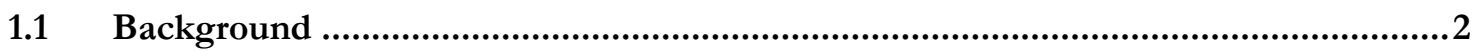

2 Modelling stochastic programming problems...........................................................

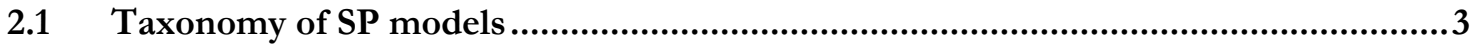

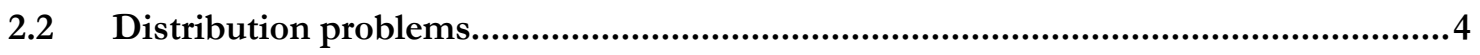

2.3 Stochastic Programming Problems with Recourse .....................................................5

2.4 Chance-Constrained Problems .................................................................................... 8

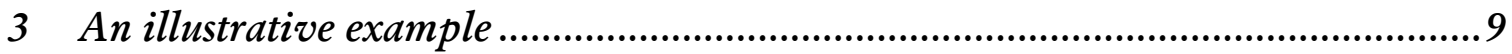

3.1 A deterministic network distribution model................................................................9

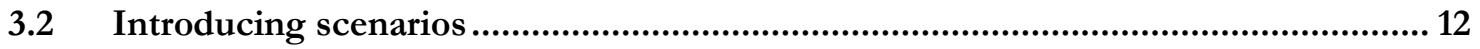

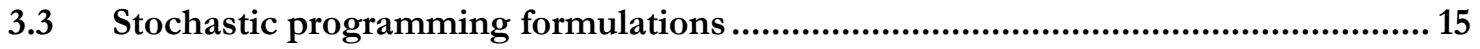

4 Adapting algebraic modelling languages for SP ........................................... 18

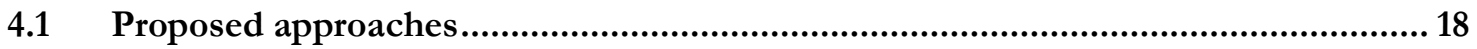

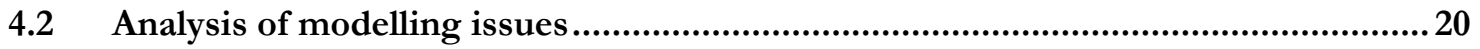

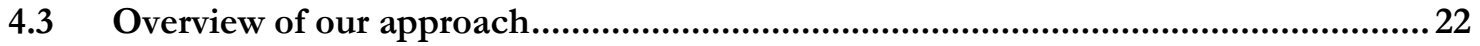

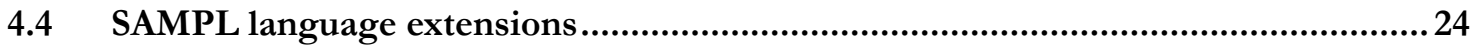

4.5 Implementation of the illustrative examples in SAMPL ........................................... 31

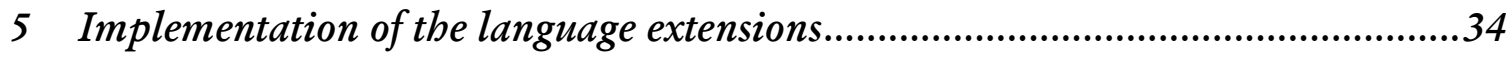

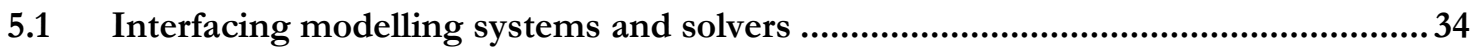

5.2 Communicating with scenario generators.................................................................. 37

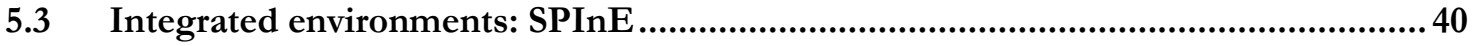

6 Conclusions and directions for future research ..............................................41

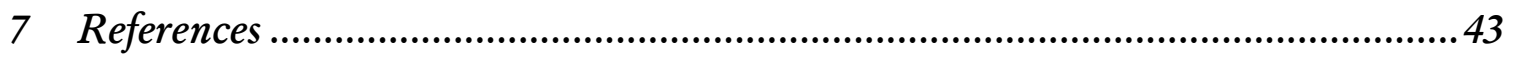




\section{Introduction}

\subsection{Background}

Over the past decades, stochastic programming models have gained wide acceptance by practitioners in the field of optimisation and operations research. Research in financial areas such as portfolio management and pension funds management (Ziemba and Mulvey 1998), in manufacturing networks and supply chain optimisation (Eppen, Martin et al. 1989; Lucas, Messina et al. 1997; Escudero, Galindo et al. 1999; Shapiro 2001), as well as in energy and environmental planning (Pereira and Pinto 1985; Bosetti, Messina et al. 2002), has demonstrated that the solution of stochastic programming models is superior to that of deterministic models in terms of expectation over future events. Indeed, stochastic programming models explicitly consider uncertainty in some of the model parameters, and provide optimal decisions which are hedged against such uncertainty.

Unfortunately, the size of stochastic programming problems grows exponentially with the number of decision stages considered by the models and with the number of possible outcomes of the random parameters at each stage. This is a challenging issue both in terms of modelling and solution algorithms. The implementation of large scale mathematical programming models implies the generation of matrices which represent the problem constraints and are stored in machine readable form in order to be interpreted by the solvers. This generation process used to be performed by adhoc software modules, and required considerable development effort. For deterministic models, the evident drawbacks of this approach were overcome with the advent of a class of declarative programming languages known as Algebraic Modelling Languages (AML), which represent powerful and flexible instruments for the generation of model data instances. The entities of the optimisation models (sets, decision variables, parameters, constraints, objectives etc.) are defined in a way that closely resembles the algebraic notation. AMPL (Fourer, Gay et al. 1993), GAMS (Brooke, Kendrick et al. 1998), AIMMS (Bisschop and Roelofs 1999) and MPL (Maximal Software 2002) are amongst the most commonly used AMLs. Most of these support the formulation of Linear Programs (LP), Mixed Integer Programs (MIP), Quadratic Programs (QP) and recently Non Linear Programs (NLP), and are capable of generating model instances using machine readable formats.

Stochastic programming models are characterised by the presence of uncertainty associated with some of their parameters, which therefore become random variables with a specified probability distribution. Unfortunately, the existing modelling languages do not provide appropriate constructs for the definition of this random nature. In this report we present a direct approach for the definition of SP models via declarative syntax, and illustrate how this idea can be applied to extend virtually any 
algebraic modelling language. We consider the AMPL systems and by means of examples, define the syntax of the stochastic extension SAMPL.

The rest of the report is organised as follows:

In section 2, we define various classes of models for optimum decision making under uncertainty. In section 3 we introduce an illustrative model which we first formulate as a linear program, and then extend into a two stage and multistage stochastic programming problem with recourse, and finally into a chance constrained problem. In section 4, we analyse the modelling requirements for stochastic programming problems and introduce our approach in extending algebraic modelling languages. We define new language constructs for AMPL and use the resulting SAMPL extension to formulate the illustrative model. In section 5 we consider the practical implementation of these stochastic programming extensions; in particular, we focus on the integration of algebraic modelling systems with scenario generators and solution methods. We conclude in section 6 with some final remarks and future research directions.

\section{Modelling stochastic programming problems}

\subsection{Taxonomy of SP models}

There is a growing body of literature covering many applications of SP (see Annals of Operations Research, 1999 vol. 85). In this section we consider the family of models which is now well established and come under the broad heading of optimum decision making under uncertainty. We follow the classification of stochastic programming problems introduced by (Gassmann and Ireland 1996). We make a small extension of their categorisation by adding the Expected Value models as a subclass of the distribution problems leading to a working taxonomy shown in Figure 1.

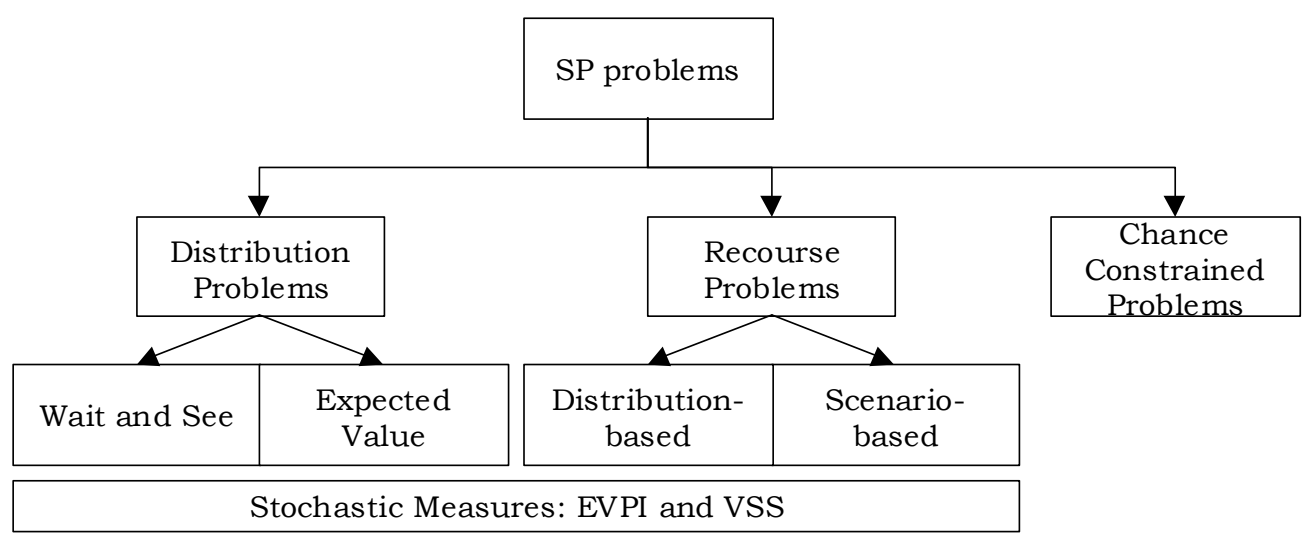

Figure 1 Taxonomy of SP problems. 
We illustrate these classes by first considering the linear programming problem:

$Z=\min c x$

subject to $A x=b$

$x \geq 0$

where $A \in R^{m \times n} ; c, x \in R^{n} ; b \in R^{m}$

Let $(\Omega, F, P)$ denote a (discrete) probability space where $\omega \in \Omega$ denote the events. Let us denote the realizations of $A, b, c$ for a given $\omega$ as:

$\left(A^{\omega}, b^{\omega}, c^{\omega}\right)=\xi^{\omega} \quad$ or $\quad \xi(\omega)$

We use $p^{\omega}=p(\xi(\omega))$ to denote the probability associated with the realisation $\xi(\omega)$. The classes of stochastic models illustrated in Figure 1 are defined in the following sections.

\subsection{Distribution problems}

The distribution of the objective function value for different realisations of the random parameters and also for the expected value of such parameters are broadly known as the distribution problems.

\section{The Expected Value Problem}

The Expected Value (EV) model is constructed by replacing the random parameters by their expected values. Such EV model is thus a linear program, as the uncertainty is dealt with before it is introduced into the underlying linear optimisation model. It is common practice to formulate and solve the EV problem in order to gain some insight into the decision problem. Let the constraint sets corresponding to the problem stated in (1) be defined as:

$F^{\omega}=\left\{x \mid A^{\omega} x=b^{\omega}, x \geq 0\right\} \quad$ for $\quad\left(A^{\omega}, b^{\omega}, c^{\omega}\right)$ or $\xi(\omega)$

We can reconsider (1) as an expected value or an average value problem where:

$(\bar{A}, \bar{b}, \bar{c})=\bar{\xi}=E\left[\xi^{\omega}\right]=\sum_{\omega \in \Omega} p^{\omega} \xi^{\omega}$

$Z_{E V}=\min \bar{c} x$

subject to

$\bar{A} x=\bar{b}$ 
Let $x^{*}{ }_{e v}$ denote the optimal solution to the above problem. This solution can be evaluated for all possible realisations $\omega \in \Omega$. We can thus determine the corresponding objective function values and compute what is called the expectation of the expected value solution:

$Z_{E E V}=E\left[c^{\omega} x_{e v}^{*}\right\rfloor$

If, however, an $\omega$ exists such that: $x^{*}{ }_{e v} \notin F^{\omega}$, i.e. $x^{*}{ }_{e v}$ is not feasible for some realisations of the random parameters, we set:

$Z_{E E V} \rightarrow+\infty$

\section{Wait and See Problems}

Wait and See (WS) problems assume that the decision-maker is somehow able to wait until the uncertainty is resolved before implementing the optimal decisions. This approach therefore relies upon perfect information about the future. Because of its very assumptions such a solution cannot be implemented and is known as the "passive approach". Wait and see models are often used to analyse the probability distribution of the objective value, and consist of a family of LP models, each associated with an individual scenario in the event tree. The corresponding problem is stated as:

$Z^{\omega}=\min c^{\omega} x$

$A^{\omega} x=b^{\omega}$

The expected value of the wait and see solutions is defined as:

$Z_{w s}=E\left[Z^{\omega}\right]=\sum_{\omega \in \Omega} Z^{\omega} p^{\omega}$

\subsection{Stochastic Programming Problems with Recourse}

A simple (single stage) stochastic programming model can be formulated as follows:

$Z_{H N}=\min E\left\lfloor c^{\omega} x\right\rfloor$

where $x \in F$

and $\quad F=\bigcap_{\omega \in \Omega} F^{\omega}$ 
The term Here and Now $(\mathrm{HN})$ is often used to refer to stochastic programming problems, reflecting the fact that decisions are taken before perfect information on the states of nature is revealed.

The classical stochastic linear program with recourse makes the dynamic nature of SP explicit, by separating the model' s decision variables into first stage strategic decisions which are taken facing future uncertainties and second stage recourse (corrective) actions, taken once the uncertainty is revealed. The formulation of the classical two-stage SP model with recourse is as follows:

$$
\begin{gathered}
Z_{H N}=\min \quad c x+E_{\omega} Q(x, \omega) \\
\text { subject to } \quad A x \quad=b \\
x \geq 0,
\end{gathered}
$$

Where:

$$
\begin{array}{ll}
Q(x, \omega)= & \min f(\omega) y(\omega) \\
\text { subject to } & D(\omega) y(\omega)=d(\omega)+B(\omega) x \\
& y(\omega) \geq 0 . \\
& \omega \in \Omega
\end{array}
$$

The matrix $A$ and the vector $b$ are known with certainty. The function $Q(x, \omega)$, referred to as the recourse function, is in turn defined by the linear program (12). The technology matrix $D(\omega)$, also known as the recourse matrix, the right-hand side $d(\omega)$, the inter-stage linking matrix $B(\omega)$, and the objective function coefficients $f(\omega)$ of this linear program are random. For a given realisation $\omega$, the corresponding recourse action $y(\omega)$ is obtained by solving the problem set out in (12).

As the future unfolds in several sequential steps and subsequent recourse actions are taken, we deal with the generalisation of the two-stage recourse problem, known as multistage stochastic programming problem with recourse. A decision made in stage $t$ should take into account all future realisations of the random parameters and such decisions only affect the remaining decisions in stages $t+1 \ldots T$. In stochastic programming this concept is known as non-anticipativity. The general formulation of a multistage recourse problem is set out in equations (13) - (15) below:

$Z_{H N}=\min _{x_{1}}\left\{c_{1} x_{1}+E_{\xi_{2}}\left[\min _{x_{2}} c_{2} x_{2}+E_{\xi_{3} \mid \xi_{2}}\left[\min _{x_{3}} c_{3} x_{3}+\ldots+E_{\xi_{T}\left|\xi_{T-1}\right| \ldots \xi_{2}} \min _{x_{T}} c_{T} x_{T}\right]\right]\right\}$

subject to: 


$$
\begin{aligned}
& A_{11} x_{1} \quad=b_{1} \\
& A_{21} x_{1}+A_{22} x_{2}=b_{2} \\
& A_{31} x_{1}+A_{32} x_{2}+A_{33} x_{3} . \quad=b_{3} \\
& \vdots \quad \ddots \ddots \quad \vdots \\
& A_{T 1} x_{1}+A_{T 2} x_{2}+A_{T 3} x_{3}+\ldots \quad+A_{T T} x_{T}=b_{T}
\end{aligned}
$$

where $t=1, . ., T$ represents the planning horizon and the vectors:

$\xi_{t}=\left(b_{t}, c_{t}, A_{t 1}, \ldots A_{t T}\right) \quad \forall \mathrm{t} \in[2, \ldots, T]$

are random variables on a probability space $(\Omega, F, P)$. It is important to stress the difference between decision stages and model time periods. Although these coincide in many applications, stage can be regarded in general as a time period where new information about the state of nature is provided, that is the realisation of the random vectors can be observed.

\section{Scenario based recourse problems}

Reconsider the random parameter vector $\xi^{\omega}$ introduced in (2) and used in the definition of the given classes of models. Given that we have considered discrete distributions, the event parameter takes the range of values $\omega=1, \ldots,|\Omega|$; there are associated probabilities $p^{\omega}$ and random parameter vector realisations $\xi^{\omega}$ such that:

$$
\sum_{\omega \in \Omega} p^{\omega}=1 \quad \text { and } \quad \Xi=\bigcup_{\omega \in \Omega} \xi^{\omega}
$$

In (16), $\Xi$ is the set of all random parameter vectors and is often called the set of scenarios. For the multistage recourse problem (13) - (15), if the probability distribution of the random parameter vectors is discrete, the uncertainty defines a random structure in the form of an event tree, which represents the possible sequence of realisations (scenarios) over the time horizon (see Figure 2). When the event tree is explicitly given, we refer to the model as a scenario based recourse problem. 


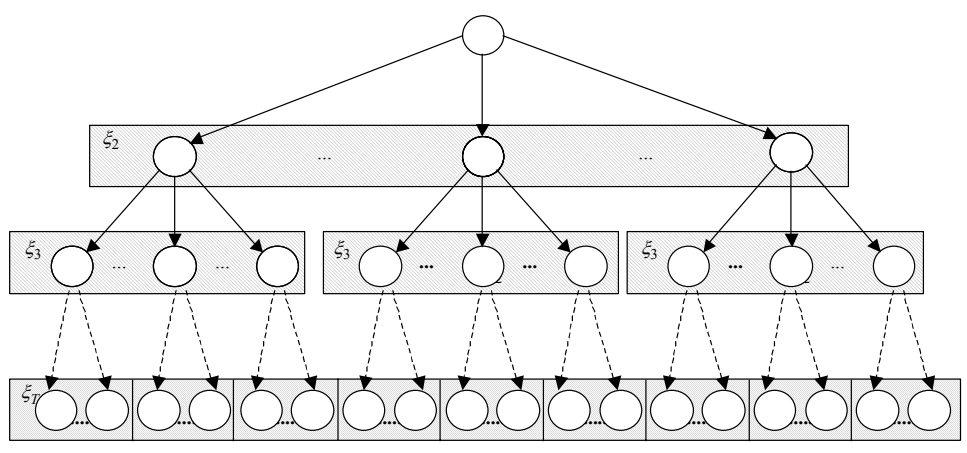

Figure 2. Event tree and scenarios

In the multistage problem (scenario based), the event tree serves two purposes:

- specify the model of randomness and

- define the algebraic model structure including hierarchal (or recursive) non anticipativity restrictions.

Taking into account the event tree, it is possible to define a scenario as the sequence of realisations $\xi_{1 \ldots} \xi_{T}$ which leads from the root of the tree to one of the leafs (Messina and Mitra 1997).

\section{Distribution based Problem}

An event tree can be also implied by defining the probability distributions of the random parameters, in which case the model is called distribution based recourse problem. Gassmann and Ireland (Gassmann and Ireland 1996) expand this concept in their work. This second class of problems, however, introduces various difficulties in the model specification using algebraic modelling languages and in terms of the solution process, in particular when some of the random parameters are continuously distributed. An approximation can be achieved by adopting appropriate sampling procedures, whereby the distributions may be replaced by an event tree.

\subsection{Chance-Constrained Problems}

Another important class of stochastic programming models is represented by the chance-constrained problems (CCP) (Charnes and Cooper 1959). These can be dynamic or static models where one or more constraints are probabilistic. The general formulation of a chance-constrained problem is: 
$Z_{C C P}=\min c x$

s.t.

$A_{0} x=b_{0}$

$P\left\{A_{i} x \geq h_{i}\right\} \geq \beta_{i} \quad i=1 . . I$,

where:

$\beta_{i} \in[0,1]$

is a reliability level and:

$\xi_{i}=\left(A_{i}, h_{i}\right) \quad \forall i=1 . . I$

is a random vector on the probability space $(\Omega, F, P)$.

If $A_{i}$ is a row vector, the $i$-th constraint is called an individual chance constraint. If $A_{i}$ is an $r \times c$ matrix with $r>1$, then the $i$-th constraint is referred to as joint chance constraint. Chance constraints can also be introduced in recourse problems, thus producing "hybrid" SP models.

\section{An illustrative example}

In this section, we use a distribution network problem to illustrate each main class of SP problems. We first provide the formulation of a linear deterministic model, which we extend to include uncertain demands. This leads to the definition of a two-stage SP model with recourse at first, followed by a multistage version of the same model. We finally introduce probabilistic constraints and obtain a chance-constrained model. In the remainder of the report, we will use these models in order to highlight the inadequacies of the current modelling languages in terms of SP modelling. Also, we will show how we can use our SP language constructs to overcome these limitations.

\subsection{A deterministic network distribution model}

We state the distribution problem as follows:

A clothing manufacturer produces goods in two factories. The manufacturer supplies its retailers with three products, Shirts, Skirts and Jeans. The products are shipped to three main dealers in quantities of tens of thousands, and the dealers can carry over inventory from one period to the next. A link or inventory variable is introduced which represents the amount of commodity transferred from one period to the next. The manufacturer knows the production costs, transportation costs, 
inventory costs for the next month with certainty. For simplicity it is assumed that all costs, production and inventory capacities are known with certainty for each time period whereas the dealer requirements can vary for each time period (e.g. for seasonal reasons). Production rates and the initial inventory of each product at each factory are known as well. A shortage penalty is introduced if the demand is not met. Figure 3 shows the interactions of the different parts of the network.

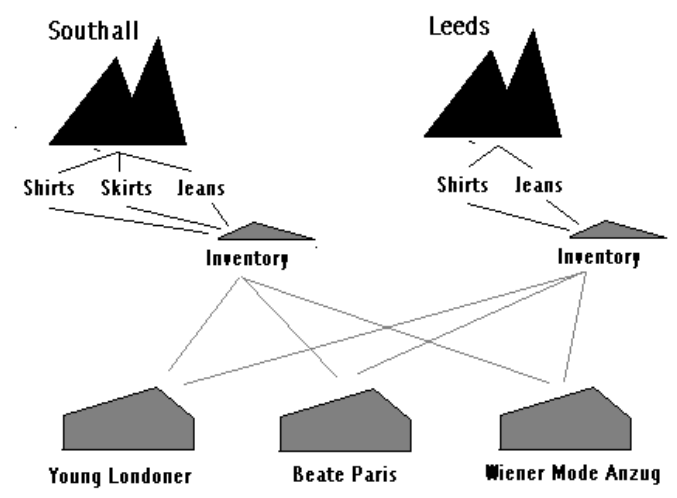

Figure 3. Physical network structure

The resulting network problem is a dynamic linear programming problem where the objective is to minimise the overall costs over the next 4 months. This problem may be formulated in a structured fashion as follows:

\section{Indices and Dimensions}

The model indices and dimensions are defined as follows:

$i=1, \ldots, N F \quad$ denotes the factories $(\mathrm{NF}=2)$

$j=1, \ldots, N P \quad$ denotes the products $(\mathrm{NP}=3)$

$k=1, \ldots, N K \quad$ denotes the dealers $(\mathrm{NK}=3)$

$t=1, \ldots, N T \quad$ denotes the time period $(\mathrm{NT}=4)$

\section{Parameters}

$q_{j i} \quad$ the cost of producing one unit of product $j$ at factory $i$

$c_{i k} \quad$ the cost of transporting one unit of any product from factory $i$ to dealer $\mathrm{k}$

$v_{j i} \quad$ the cost for holding one unit of product $j$ at factory $i$ in inventory

$p_{j k} \quad$ the unit cost for not meeting demand for product $\mathrm{j}$ at dealer $\mathrm{k}$

$a_{j i} \quad$ the production capacity for product $\mathrm{j}$ at factory $\mathrm{i}$ 
$n_{j i} \quad$ the inventory capacity of product $\mathrm{j}$ at factory $\mathrm{i}$

$d_{j k t} \quad$ the requirement of dealer $\mathrm{k}$ for product $\mathrm{j}$ at time period $\mathrm{t}$

$l_{j i} \quad$ the initial inventory of product $\mathrm{j}$ at factory $\mathrm{i}$

\section{Decision variables}

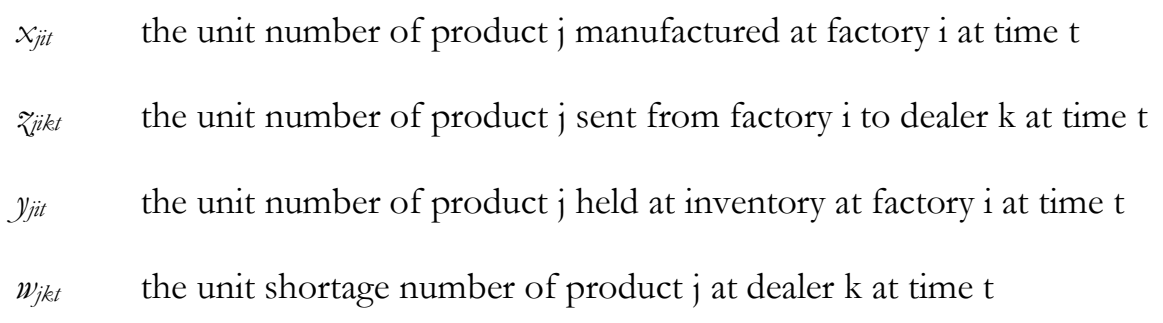

\section{Objective function}

The manufacturers aim is to minimise the overall costs over the planning horizon.

$$
\begin{aligned}
& \text { Min Costs }=\text { Total_Production_Cost } \quad+\text { Total_Transportation_Cost }+ \\
& \text { Total_Inventory_Cost } \quad+\text { Total_Shortage_Cost } \\
& \text { Min Costs }=\sum_{j=1}^{N P} \sum_{i=1}^{N F} \sum_{t=1}^{N T} q_{j i} \cdot x_{j i t}+\sum_{j=1}^{N P} \sum_{i=1}^{N F} \sum_{k=1}^{N K} \sum_{t=1}^{N T} c_{i k} \cdot z_{j i k t} \\
& +\sum_{j=1}^{N P} \sum_{i=1}^{N F} \sum_{t=1}^{N T} v_{j i} \cdot y_{j i t}+\sum_{j=1}^{N P} \sum_{k=1}^{N K} \sum_{t=1}^{N T} p_{j k} \cdot w_{j k t}
\end{aligned}
$$

\section{Constraints}

Satisfying dealer requirements at every time period

$$
\sum_{i=1}^{N F} z_{j k t}+w_{j k t}=d_{j k t} \quad \forall j, k, t
$$

Inventory balance at time period $t=1$

$$
x_{j i t}+l_{j i}=y_{j i t}+\sum_{k=1}^{N K} z_{j i k t} \quad \forall j, i \text { and } t=1
$$

Inventory balance for $t>1$

$$
x_{j i t}+y_{j i t-1}=y_{j i t}+\sum_{k=1}^{N K} z_{j i k t} \quad \forall j, i \quad \text { and } \quad t=2, \ldots T
$$


Inventory capacity constraint

$y_{j i t} \leq n_{j i} \quad \forall j, i, t$

Production capacity constraint

$x_{j i t} \leq a_{j i} \quad \forall j, i, t$

Non-negativity of decision variables

$x_{j i t} \geq 0, z_{j i k t} \geq 0, y_{j i t} \geq 0, w_{j k t} \geq 0 \quad \forall j, i, k, t$

\subsection{Introducing scenarios}

The model formulated above assumes that all parameters are known with certainty. In this SP example, we consider the demand as a random parameter characterized by known probability distribution. The realizations of the uncertain parameters can be provided either via probability distributions or in the form of scenario trees. In this example, we use a scenario tree which represents the demand for each product. A simple generation procedure for these scenarios is shown in Figure 4: given an initial value for the demand, the rule takes into account two possible realisations of the demand in each time period $t>1$. The percentage at each node represents the change of the demand from the value at the parent node. The resulting scenario tree consists of a set of scenarios $\Xi=[1,2 . ., 8]$. At each node, the probabilities for the two possible realisation are equal $(\operatorname{Pr}=0.5)$, thus the resulting scenarios are equiprobable with probability $\operatorname{Pr}[s]=0.5 * 0.5 * 0.5=0.125$. The demand parameter is defined as:

$d_{j k t s} \quad$ is the requirement of dealer $\mathrm{k}$ for product $\mathrm{j}$ at time period $\mathrm{t}$ under scenario $\mathrm{s}$. 


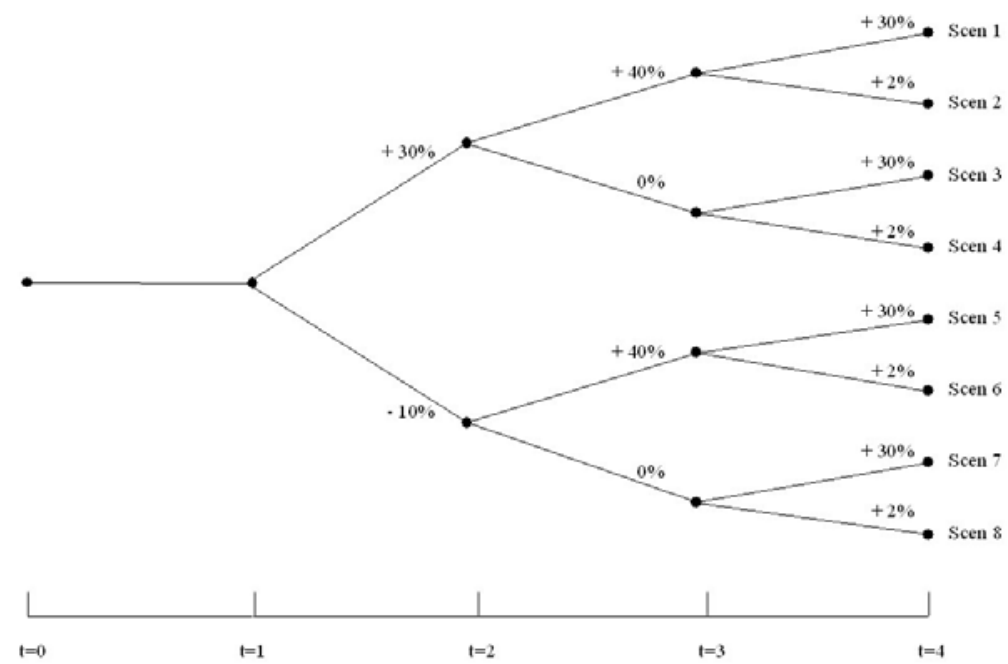

Figure 4. Scenario generator for the demand.

The decisions made at $t=1$ need to take into account the future uncertainty of the demand. The (scenario dependent) corrective actions are taken in $t>1$.

We formulate the deterministic equivalent model of the recourse problem by making a copy of the problem for each scenario and then tying the copies together by requiring certain variables to be equal according to the structure of the underlying scenario tree. This formulation is called the splitvariable representation and enables us to define a two-stage version and a multistage version of the same problem by simply adding or relaxing non-anticipativity constraints. The model entities can be summarised as follows:

\section{Indices and dimensions}

$\begin{array}{lll}i=1, \ldots, N F & \text { denotes the factories } & (N F=2) \\ j=1, \ldots, N P & \text { denotes the products } & (N P=3) \\ k=1, \ldots, N K & \text { denotes the dealers } & (N K=3) \\ t=1, \ldots, N T & \text { denotes the time period } & (N T=4) \\ s=1, \ldots, N S & \text { denotes the scenarios } & (N S=8)\end{array}$

\section{Parameters}

$q_{j i} \quad$ the cost of producing one unit of product $j$ at factory $i$

$c_{i k} \quad$ the cost of transporting one unit of any product from factory $\mathrm{i}$ to dealer $\mathrm{k}$

$v_{j i} \quad$ the cost for holding one unit of product $j$ at factory $i$ on inventory

$p_{j k} \quad$ the shortage penalty for product $\mathrm{j}$ at dealer $\mathrm{k}$ 
$a_{j i} \quad$ the production capacity of product $j$ at factory $i$

$n_{j i} \quad$ the inventory capacity of product $\mathrm{j}$ at factory $\mathrm{i}$

$d_{j k s s} \quad$ the requirement of dealer $\mathrm{k}$ for product $\mathrm{j}$ at time period $\mathrm{t}$ under scenario $\mathrm{s}$

$l_{j i} \quad$ the initial inventory of product $\mathrm{j}$ at factory $\mathrm{i}$

$\pi_{s} \quad$ the probability for scenario s

\section{Decision variables}

$x_{j i t s} \quad$ the unit number of product $j$ manufactured at factory $i$ at time $t$, scenario $s$,

$z_{i j k t s}$ the unit number of product $\mathrm{j}$ sent from factory $\mathrm{i}$ to dealer $\mathrm{k}$ at time $\mathrm{t}$, scenario $\mathrm{s}$,

$y_{j i t s} \quad$ the unit number of product $j$ held at inventory at factory $i$ at time $t$, scenario $s$,

$w_{j k t s} \quad$ the unit shortage number of product $\mathrm{j}$ at dealer $\mathrm{k}$ at time $\mathrm{t}$, scenario $\mathrm{s}$.

The objective of the recourse problems is to minimise the the expectation of the total costs over all scenarios.

\section{Objective function}

$$
\begin{aligned}
\text { Min Costs }=\sum_{s=1}^{N S} \pi_{s} & \cdot\left(\sum_{j=1}^{N P} \sum_{i=1}^{N F} \sum_{t=1}^{N T} q_{j i} \cdot x_{j i t s}+\sum_{j=1}^{N P} \sum_{i=1}^{N F} \sum_{k=1}^{N K} \sum_{t=1}^{N T} c_{i d} \cdot z_{j i k t s}\right. \\
& \left.+\sum_{j=1}^{N P} \sum_{i=1}^{N F} \sum_{t=1}^{N T} v_{j i} \cdot y_{j i t s}+\sum_{j=1}^{N P} \sum_{k=1}^{N K} \sum_{t=1}^{N T} p_{j k} \cdot w_{j k t s}\right)
\end{aligned}
$$

\section{Constraints}

All constraints need to be satisfied for all scenarios, hence the model's constraints are given by:

Satisfying dealer requirements at every time period and every scenario:

$\sum_{i=1}^{N F} z_{j k t s}+w_{j k t s}=d_{j k t s}$

$\forall j, k, t, s$

Inventory balance at time period $\mathrm{t}=1$ :

$x_{j i t s}+l_{j i}=y_{j i t s}+\sum_{k=1}^{N K} z_{j i k t s}$

$\forall j, i, s \quad$ and $\quad t=1$

Inventory balance for $\mathrm{t}>1$ : 
$x_{j i t s}+y_{j i t-1 s}=y_{j i t s}+\sum_{k=1}^{N K} z_{j i k t s}$

Inventory capacity constraint:

$y_{j i t s} \leq n_{j i}$

Production capacity constraint:

$x_{j i t s} \leq a_{j i}$

Non-negativity of decision variables:

$x_{j i t s} \geq 0, z_{j i k t s} \geq 0, y_{j i t s} \geq 0, w_{j k t s} \geq 0$ $\forall j, i, s \quad$ and $\quad t=2, \ldots T$

$\forall j, i, t, s$

$\forall j, i, t, s$

$\forall j, i, k, t, s$

\subsection{Stochastic programming formulations}

\section{The two-stage stage recourse problem}

The problem stated in the previous section is the formulation of the Wait and See model: each scenario is independently optimised. The two-stage recourse model requires the first stage decision variables (for $t=1$ ) to be the same for all scenarios. This can be achieved by introducing a set of nonanticipativity constraints. We recall from section 3.2 that $\Xi=[1,2 . ., 8]$, hence the restrictions can be written as:

$$
\begin{array}{ll}
x_{j i 1 s}=x_{j i 1 s^{\prime}} & \forall j, i \text { where } s \in[1 . .7], \text { and } s^{\prime}=s+1 \\
y_{j i 1 s}=y_{j i 1 s^{\prime}} & \forall j, i \text { where } s \in[1 . .7], \text { and } s^{\prime}=s+1 \\
w_{j k 1 s}=w_{j k 1 s^{\prime}} & \forall j, i \text { where } s \in[1 . .7], \text { and } s^{\prime}=s+1 \\
z_{j i k 1 s}=z_{j i k 1 s^{\prime}} & \forall j, i, k \text { where } s \in[1 . .7], \text { and } s^{\prime}=s+1
\end{array}
$$

These constraints impose on the model the scenario tree structure shown in Figure 5. 


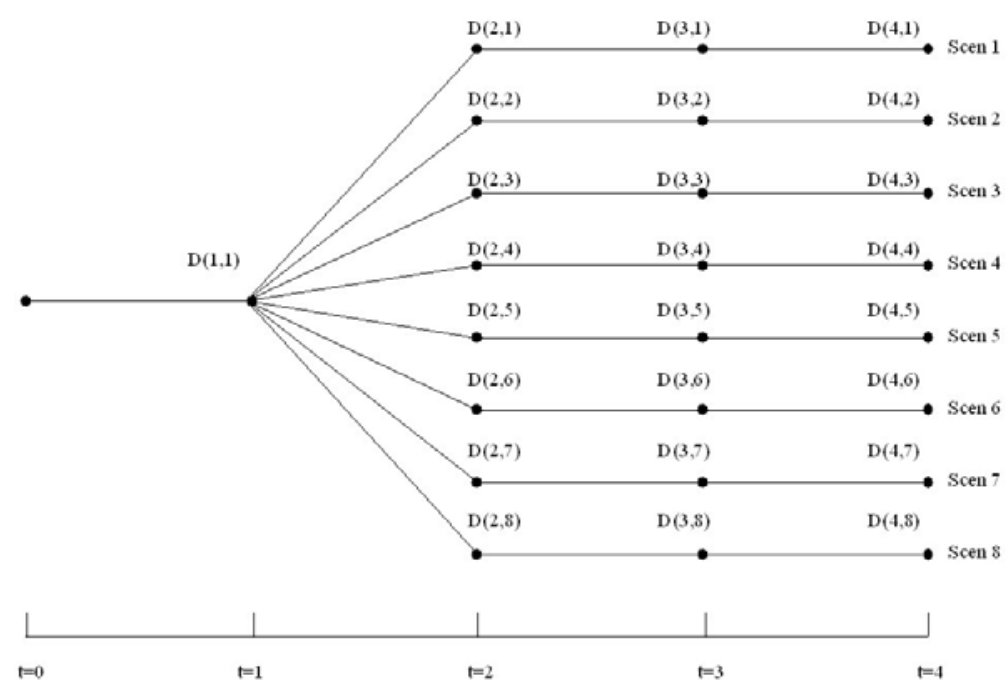

Figure 5. Two-stage scenario tree.

Considering the sets of variables:

$\bar{x}_{t s}=\left\{x_{j i t s} \forall j, i\right\}$

$\bar{y}_{t s}=\left\{x_{j i t s} \forall j, i\right\}$

$\bar{w}_{t s}=\left\{w_{j k t s} \forall j, k\right\}$

$\bar{z}_{t s}=\left\{z_{j i k t s} \forall j, i, k\right\}$

we can rewrite the above constraints in shorter notation:

$D_{11}=(\bar{x}, \bar{y}, \bar{w}, \bar{z})_{t s}=(\bar{x}, \bar{y}, \bar{w}, \bar{z})_{t s^{\prime}} \quad t=1$ where $s \in[1 . .7]$ and $s^{\prime}=s+1$

The non anticipativity forces these sets of decisions to be the same; we can thus substitute them with a named set $D_{11}$, which represents the decisions associated with the (only) node in the first stage of the scenario tree. In the two stage model, each scenario is independent for $t>1$. The decisions to be taken in the remaining nodes of the tree can be hence defined as:

$D_{t s}=(\bar{x}, \bar{y}, \bar{w}, \bar{z})_{t s} \quad t>1, s \in \Xi$

If we use the non-anticipativity constraints to eliminate by substitution the variables that are forced to be the same, we obtain what is often referred to as the compact or implicit deterministic equivalent representation. 


\section{The multistage stage recourse problem}

In order to formulate the multi-stage stochastic linear program for the given problem we assume scenarios sharing the same history up to a stage $t$ in the scenario tree, must also share the same decisions up to that stage. This means that more non-anticipativity constraints need to be added, so that the tree structure given in Figure 4 can be embodied into the model. The full set of nonanticipativity constraints, expressed in short notation, is set out below:

$$
\begin{array}{ll}
D_{11}=(\bar{x}, \bar{y}, \bar{w}, \bar{z})_{1 s}=(\bar{x}, \bar{y}, \bar{w}, \bar{z})_{1 s^{\prime}} & \text { where } s \in[1 . .7] \text { and } s^{\prime}=s+1 \\
D_{21}=(\bar{x}, \bar{y}, \bar{w}, \bar{z})_{2 s}=(\bar{x}, \bar{y}, \bar{w}, \bar{z})_{2 s^{\prime}} & \text { where } s \in[1 . .3] \text { and } s^{\prime}=s+1 \\
D_{22}=(\bar{x}, \bar{y}, \bar{w}, \bar{z})_{2 s}=(\bar{x}, \bar{y}, \bar{w}, \bar{z})_{2 s^{\prime}} & \text { where } s \in[5.7] \text { and } s^{\prime}=s+1 \\
D_{31}=(\bar{x}, \bar{y}, \bar{w}, \bar{z})_{3 s}=(\bar{x}, \bar{y}, \bar{w}, \bar{z})_{3 s^{\prime}} & \text { where } s=1 \text { and } s^{\prime}=2 \\
D_{32}=(\bar{x}, \bar{y}, \bar{w}, \bar{z})_{3 s}=(\bar{x}, \bar{y}, \bar{w}, \bar{z})_{3 s^{\prime}} & \text { where } s=3 \text { and } s^{\prime}=4 \\
D_{33}=(\bar{x}, \bar{y}, \bar{w}, \bar{z})_{3 s}=(\bar{x}, \bar{y}, \bar{w}, \bar{z})_{3 s^{\prime}} & \text { where } s=5 \text { and } s^{\prime}=6 \\
D_{34}=(\bar{x}, \bar{y}, \bar{w}, \bar{z})_{3 s}=(\bar{x}, \bar{y}, \bar{w}, \bar{z})_{3 s^{\prime}} & \text { where } s=7 \text { and } s^{\prime}=8
\end{array}
$$

in the last stage, a distinct decision is taken for each scenario, therefore we define:

$$
D_{4 s}=(\bar{x}, \bar{y}, \bar{w}, \bar{z})_{t s} \quad t=4, s \in \Xi
$$

The resulting decision tree is shown in Figure 6.

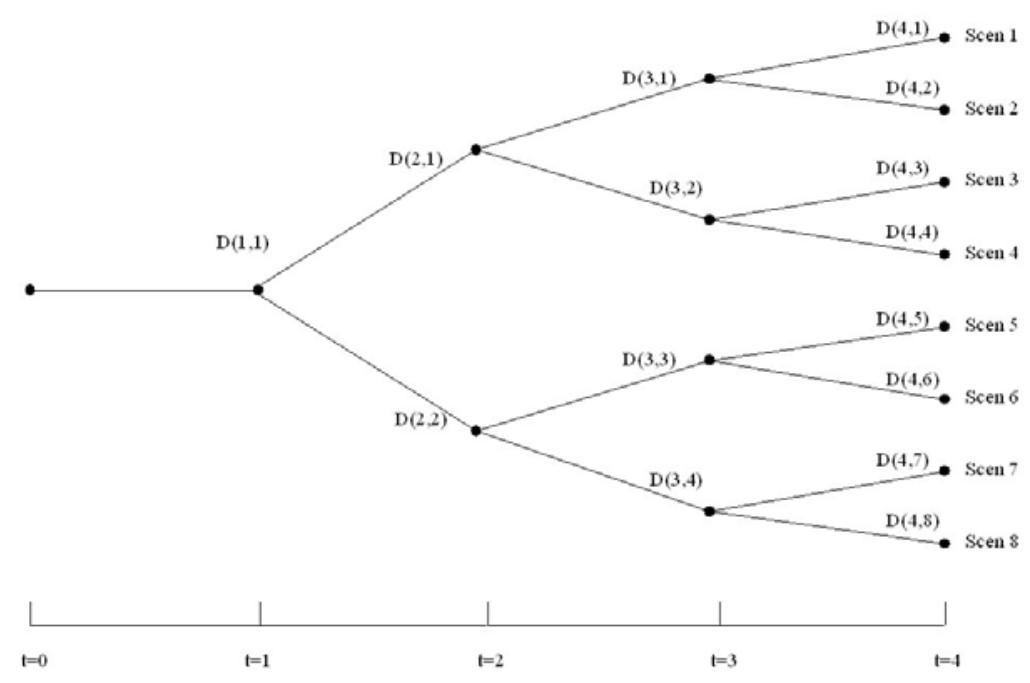

Figure 6. Multistage scenario tree. 


\section{Introducing chance constraints into the model}

We can introduce a probabilistic constraint into the model by making the following assumptions:

- the demand is a random parameter, with probability distribution expressed via the event tree of Figure 4.

- No shortage is allowed at the dealer nodes, but demand needs to be satisfied with at least a given probability.

The chance constraint can be now stated as follows: the probability of satisfying dealer requirements at every time period must be greater than a given $\beta$.

$$
P\left(\sum_{i=1}^{N F} z_{j i k t s} \geq d_{j k t s}\right) \geq \beta_{j k t} \quad \forall j, k, t
$$

In our example, the probability distribution for the demand is discrete. This probabilistic constraint can be thus embodied into the model by introducing a lower bound on the number of scenarios for which the underlying linear constraint needs to be satisfied, leading to a mixed integer programming formulation (Valente 2002).

\section{Adapting algebraic modelling languages for SP}

\subsection{Proposed approaches}

Lately, practitioners in stochastic programming and experts in modelling systems have joined forces in the search for modelling language constructs, which would enable management science and operational research analysts to capture the random nature of stochastic programming models in a natural and effective way. For instance, in (Gassmann and Ireland 1995), the authors addresses the problem of defining scenario based recourse problems using a non-redundant formulation. Scenarios are specified parametrically and the scenario data can either be imported or ideally computed by the AMPL modelling system. The scenario tree structure is represented by first defining a base scenario, containing essentially the underlying deterministic multiperiod model. Additional scenarios sharing at least the root node with the base scenario are characterised by a parent scenario and the first stage in which the scenario differs from its parent. Each new scenario represents a sub-problem, with its own decisions and constraints. The non anticipativity restrictions are thereby captured implicitly.

In (Fourer 1996), the author proposes some extensions to the AMPL modelling language. These extensions should enable the definition of a stochastic programming problem with recourse in terms of a multistage (deterministic) model, a tree of data scenarios for the model, and a stochastic 
framework to specify the stages and optionally the scenarios and objective. New language constructs such as scenario and stochastic are introduced to enable the definition of a scenario as a collection of data and to declare the partition of an underlying time horizon into stages. Scenarios can be solved individually or as a recourse problem, provided that an appropriate expected value objective is defined. The authors also hint at the possibility of using a new keyword, namely random, to assign a probability distribution to given parameters, thus enabling the definition of distribution-based stochastic programming models.

In (Gassmann and Ireland 1996), the authors also propose extension to the AMPL modelling language, mainly for the definition of probability distributions of the random parameters. Again, a language construct random is introduced in distribution-based recourse problems to identify the random parameters and the variables which depend on these. The same keyword is used in chance constraint problems, along with the keywords prob and jointprob which are used to declare individual and joint chance (probabilistic) constraints respectively. Scenario-based recourse problems are dealt with as in the aforementioned work by the same authors (Gassmann and Ireland 1995). A special set, called timeset, is defined in all multistage recourse problems and identifies the problem's stages. An expectation operator is also proposed to express the expected value of random parameters in distribution-based models. Finally, the authors propose a number of keywords to indicate specific probability distributions such as discrete, normal and uniform.

In (Entriken 2001), two additional syntactical items for modelling languages are presented, namely a random construct for the definition of random parameters, and a relational operator which indicates precedence between random events. The main idea behind this approach is the fact that stochastic programming models may be seen as control theory problems, where the random events are assumed to be input to the system along with the control variables, so that at a given $t$, only the past outcomes are known, together with the distribution of the future random parameters. The author uses the syntax of the AMPL language to declare the underlying linear program, and proposes some new constructs for the uncertainty. Random parameters are declared using a random qualifier, and are assumed indexed over the set of outcomes. The importance of the time sequencing of decisions and events is highlighted and more assumptions (time conventions) are introduced to ensure the correct order. Also, the relational operators $\mid>$ and $<\mid$ are proposed, which provide partial ordering of the outcomes of the random parameters.

An alternative and innovative approach to modelling stochastic linear programming problems is presented in (Buchanan, McKinnon et al. 2001). In the paper, the authors define a language called sMAGIC, which enables the recursive definition of models which contain other (sub) models. Recursive definition is typical of Dynamic Programming and enables the preservation of the 
underlying Markov structure, which also characterises many multistage stochastic programming models. The event tree for models with a Markov structure is compactly represented via a special direct acyclic graph, which the authors call Model Link Graph (MLG). The nodes of the MLG represent model or sub-model instances, and arcs connect each model with its nested sub-models. Other work on extending algebraic modelling languages to support the formulation of stochastic programming problems include (Gay 2001), (Lopes and Fourer 2003).

\subsection{Analysis of modelling issues}

Our illustrative example introduced in section 3 highlights the difficulties of using existing algebraic modelling languages to formulate SP. This is mainly due to the lack of constructs for the definition of the randomness of the model coefficients and the scenario tree structure. A stochastic programming model can be considered as a linear programming model extended and refined by the introduction of uncertainty (see Figure 7). More precisely, the underlying LP optimisation model is extended by taking into account the probability distribution of the model's random parameters. Such distributions are provided by the models of randomness used in scenario generators, which are specific to the particular optimisation problems under investigation.

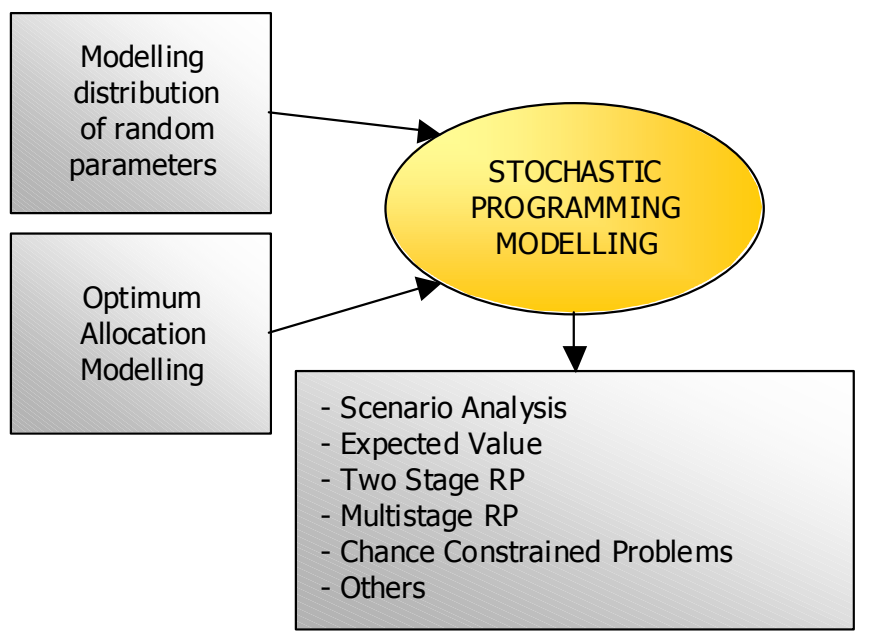

Figure 7. The combined paradigm

In general, different categories of stochastic programming problems require different language features to express the random nature of the problem. We call stochastic framework the information represented by these constructs. 


\section{Scenario based recourse problems}

The first requirement for the formulation of a stochastic programming problem using algebraic modelling languages is the declaration of the random parameters. In scenario based recourse problems, the realisations of such parameters are explicitly given in the form of a scenario tree. Each scenario is also associated with a corresponding weight (or probability). In turn, the scenario tree structure is declared in terms of stages. The stages identify the sequence of decisions in the dynamics of the underlying core model. If the temporal dimension is introduced into the model using a specific time set, the stages can be declared as subsets of this set. To summarise, the stochastic framework for scenario based recourse problems requires constructs for the definition following entities:

\begin{tabular}{|l|l|}
\hline Entities & Language Requirements \\
\hline stages information & assignment of variables and constraints to stages. \\
\hline scenarios information & $\begin{array}{l}\text { scenarios set } \\
\text { tree structure } \\
\text { scenario probabilities }\end{array}$ \\
\hline random parameters & $\begin{array}{l}\text { declaration of the random parameters in terms of the scenario } \\
\text { set. }\end{array}$ \\
\hline
\end{tabular}

Table 1. Requirements for scenario based recourse problems

\section{Modelling distribution based recourse problems}

A number of researchers have proposed extensions to algebraic languages for the formulation of such class of problems (Fourer 1996; Gassmann and Ireland 1996; Fourer 2001; Gay 2001).

In this work, we focus on the class of scenario-based recourse problems and only outline the requirements for distribution-based models. Distribution based recourse problems rely on the declaration of probability distributions for the random parameters. This can be discrete or continuous. If all random parameters are characterised by discrete distributions, the scenario tree is implied by the joint realisations of the random parameters. If one or more distributions are continuous, then there are infinite possible outcomes for the random parameters and the tree structure must be sampled from the joint distributions. The stochastic framework for distribution based problems requires constructs for the definition of:

\begin{tabular}{|l|l|}
\hline Entities & Language Requirements \\
\hline stages information & assignment of variables and constraints to stages. \\
\hline random parameters & declaration of the probability distributions associated with the \\
\hline
\end{tabular}


random parameters.

Table 2. Requirements for distribution based recourse problems

\section{Modelling chance constraints}

Chance constraints can be added to scenario-based problem or distribution based problems. For chance constrained models the stochastic framework should identify:

\begin{tabular}{|l|l|}
\hline Entities & Language Requirements \\
\hline individual chance constraints & $\begin{array}{l}\text { declaration of the individual chance constraints with related } \\
\text { reliability level. }\end{array}$ \\
\hline joint chance constraints & $\begin{array}{l}\text { declaration of the joint chance constraints with relating } \\
\text { reliability level }\end{array}$ \\
\hline random parameters & $\begin{array}{l}\text { if distribution-based, declaration of the probability distributions } \\
\text { associated with the random parameters } \\
\text { if scenario-based, declaration of the random parameters in } \\
\text { terms of scenarios }\end{array}$ \\
\hline
\end{tabular}

Table 3. Requirements for chance constrained problems

\subsection{Overview of our approach}

Ideally, a modelling language for stochastic programming problems should include a set of constructs which allow the modeller to capture the effects introduced by the uncertainty on the underlying model structure. We present a generic approach of expanding AMLs based on the concepts of underlying deterministic model and stochastic framework. The underlying deterministic model is formulated using the standard constructs provided by algebraic modelling languages. Using a set of new constructs, the modeller then declares the stochastic framework, which links the underlying deterministic model with the model of randomness. More specifically, the underlying deterministic model represents a family of independent (wait and see) models, while the stochastic framework imposes the non anticipativity implied by the structure of the scenario tree. The resulting model is the formulation of the stochastic programming recourse problem. Chance constraints can also be introduced using specific constructs.

\section{The underlying deterministic}

In a typical stochastic programming problem, it is always possible to identify an underlying deterministic model (also called the core model). The underlying deterministic model captures the 
logical structure of the problem as well as the dynamical relations within decision variables, their bounds and the objective function.

In our approach, we construct the core model in such a way that this is parametric in the dimension of scenarios. All variables and constraints are indexed over the scenarios (which are the element of a special set declared in the stochastic framework), and the objective function is the expected value over all scenarios of the individual objectives. As previously mentioned, this formulation is equivalent to a family of wait and see models.

\section{Declaration of the stochastic framework}

As we have shown in section 4.2, the stochastic framework depends on the type of stochastic programming model which is being developed. For instance, scenario based recourse problems require the explicit declaration of the scenario tree structure, while in a chance constrained problem the framework includes information on one or more probabilistic constraints. Finally, in a distribution based recourse problem, the AML should provide a set of constructs for the definition of probability distributions.

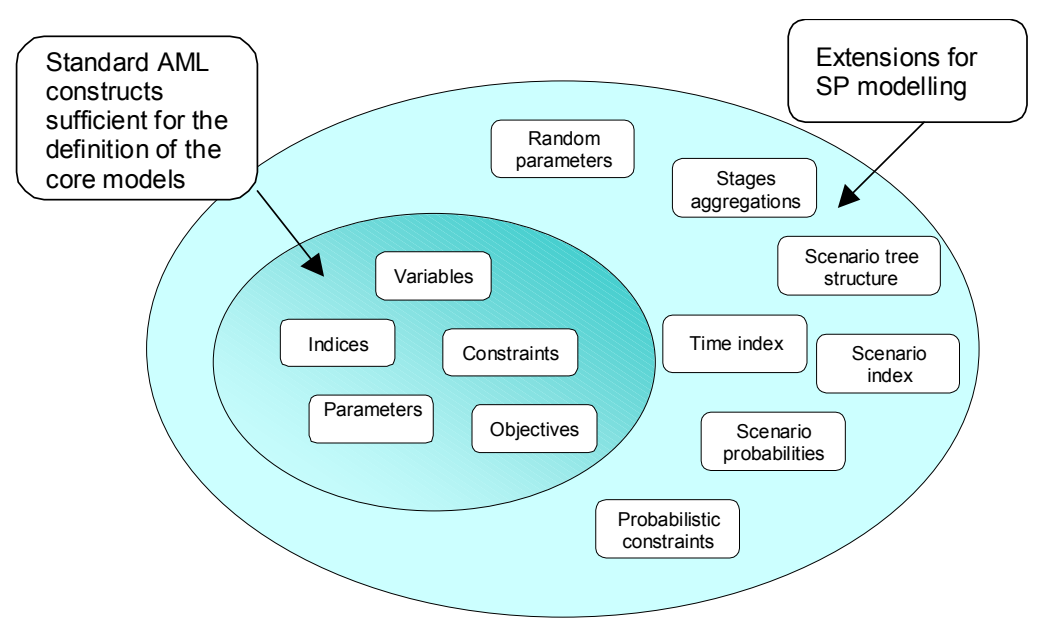

Figure 8. Extended language constructs

Figure 8 shows how the basic constructs of a modelling language for linear programming are extended to capture the stochastic framework. The definition of the new constructs is adapted to be consistent with the grammar of the underlying modelling language. We have successfully applied this approach to the AMPL language, although the same ideas can be adapted to virtually any other AML (see for instance (Valente, Mitra et al. 2001) for an application to MPL). The syntax of the extended language constructs for AMPL (which we call SAMPL) is defined in the next section. 


\subsection{SAMPL language extensions}

Taking into account the requirements expressed in the section 4.2, we have designed a set of new constructs for the AMPL language, which support the declaration of the stochastic framework information.

\section{Stages}

The assignment of variables and constraints to the different stages of the dynamic recourse model is essential in the definition of stochastic programming recourse models.

A generic approach considers the existence of a time set, which is used as an index for all variables of the model. Then a partition of such time set can be given, so that several time periods (and the variables which relate to these time periods) can be grouped into a single stage. We have implemented this approach in extending the MPL modelling language (Valente, Mitra et al. 2001). However, the fact that modellers are forced to introduce the time dimension for all variables could result in a very unnatural modelling. This is particularly true in two stage models, where the first stage and second stage decisions are usually defined using different vectors, and hence already "distinguished" from one another.

An alternative and more flexible approach is viable using modelling languages (such as AMPL), which enable the definition of suffixes. A suffix can be considered as a generic property of a variable or constraint, and can be used for our purpose to declare the stage which a variable belongs to. The stage of the constraints is determined by the stage of the variables which appear in it. The highest stage of any of such variables is the stage of the constraint.

The syntax for the assignment of a stage number to a variable is very similar to that of AMPL for other suffixes, but makes use of a predefined suffix called stage:

suffix stage IN;

let indexingopt name.stage :=expr;

Alternatively, AMPL enables the suffix to be given in the variable declarations:

var name aliasopt indexingopt attributesopt, suffix stage expr;

In the example distribution model, variables are indexed over a time set. The staging for the twostage problem can be expressed as:

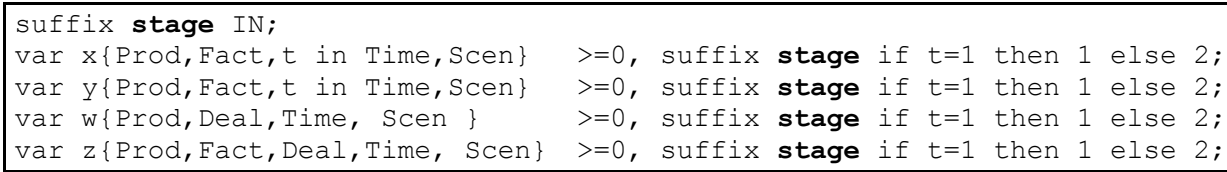


Figure 9 shows the aggregation of time periods into stages for the two-stage distribution model.

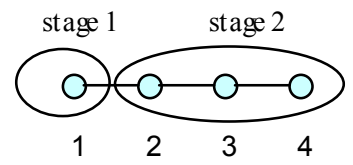

Figure 9. Two-stage aggregation.

In the multistage model, the stage of a variable is simply given by the time period which the variable belongs to:

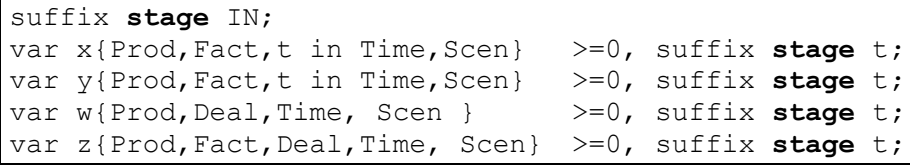

Figure 10 shows the aggregation of time periods into stages for the multistage distribution model.

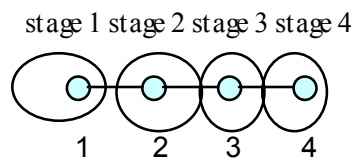

Figure 10. Multistage aggregation.

\section{Scenarios information}

Information about the scenario tree which characterises scenario-based recourse problems is provided by the modeller via a set of new keyword, which describe below:

\section{Scenario set}

In scenario-based recourse problems, the uncertainty represented by the random parameters introduces a new dimension, identified by the scenario set. This set needs to be explicitly identified, because the random parameters are indexed over it. The syntax used for the declaration of the scenario set follows the syntax of AMPL for sets, but uses scenarioset instead of set in the declaration:

scenarioset name aliasopt indexingopt attribsopt ;

Considering the example model given in section 3 , the scenario set is declared as:

param NS: $=8$

\#stochastic framework

scenarioset $\mathrm{SC}:=1 . \mathrm{NS}$; 


\section{Scenarios probabilities}

The probability distribution of the scenarios is declared using a special parameter vector, whose elements are the weights of the individual scenarios. The parameter is therefore indexed over the scenario set. The syntax used is similar to the AMPL syntax for the declaration of the other parameters, but uses the probability keyword instead of (or before) the param keyword. The syntax is the following:

probability paramopt name aliasopt indexingopt attributesopt ;

Example:

scenarioset $\mathrm{SC}:=1 \ldots \mathrm{NS}$;

probability param $\operatorname{Pr}\{\mathrm{SC}\}:=1 / \operatorname{card}(\mathrm{SC})$;

\section{Scenario Tree}

The tree keyword is used to define the scenario tree structure. In general, this structure can be considered as a set of pairs (scenario,stage), which identify the branches (and therefore the nodes) of the tree. Our language constructs enable the modeller to specify "well structured" trees in a concise way. The syntax is as follows:

tree name:= ${ }_{\text {opt }}$ tree_declaration ;

where tree_declaration is one of:

bundle list |

tlist $\mid$

$n w a y\{n\} \mid$

multibranch $\left\{n_{1}, n_{2}, \ldots, n_{S T}\right\}$ |

binary |

twostage $\{n s\}$ opt ;

\section{- bundles}

A bundle list is defined as:

bundle_list : bundles $\{$ Bundle-1, Bundle-2,.. Bundle-n\} ;

where:

Bundle-x: (stage $\left.{ }_{x}, \operatorname{scen}_{x}\right)$;

A bundle is associated to each node of the tree and consists of the stage in which the node lies, and a scenario number defined as the minimum of the ordinal values associated with the scenarios which pass through the same node (King 1994). Considering the tree structure of Figure 11, its bundles representation is formulated as: 
$(1,1)$,

$(2,1),(2,4),(2,6)$

$(3,1),(3,4),(3,6),(3,7)$,

$(4,1),(4,2),(4,3),(4,4),(4,5),(4,6),(4,7)(4,8),(4,9)$

\} ;

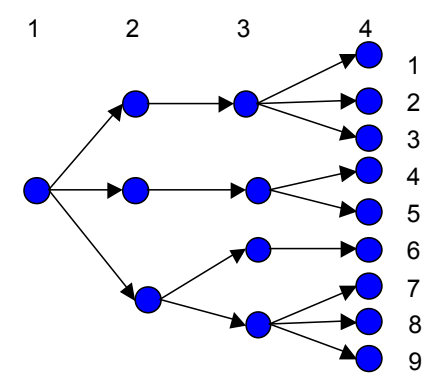

Figure 11. Example of asymmetric tree.

- tlist

An very compact representation of asymmetric trees can be obtained if the following assumption holds:

Scenarios are indexed in an order that ensures complete separation of all nested sub-trees (i.e. the scenario paths never cross one another)

In this case, a scenario tree can be represented as a list of $\mathrm{S}$ elements (tlist) whose values are the stage numbers where a scenario branches from its father scenario. A thist can thus be constructed by considering the column number associated to the leftmost element of each row of the tree matrix. The syntax for the tlist tree is the following:

tlist: $\operatorname{tlist}\left\{n 1, n 2, \ldots n_{S}\right\}$;

As an example, the tlist representing the scenario tree of Figure 11 is set out below:

tree theTree: $=$ tlist $\{1,4,4,2,4,2,3,4,4\}$;

In many applications, however, the scenario tree is characterised by a "standard" structure: the following keywords simplify the definition of such trees:

- $\operatorname{nway}\{\mathrm{n}\}$;

Defines a tree where a constant number $n$ of branches occur at each stage $\sigma=1 . . S T-1$, where ST is the number of stages in the model. The total number of scenarios $S$ of the problem is hence determined by the following expression:

$S=n^{S T-1}$ 
This must be consistent with the cardinality of the scenario set defined using the scenarioset keyword.

- $\operatorname{multibranch}\left\{\mathrm{n} 1, \mathrm{n} 2, \ldots, \mathrm{n}_{\mathrm{ST}}\right\}$;

Defines a tree where at each stage $\sigma=1 . . S T-1$ is associated a number of branches $n_{\sigma}$. The NWAY tree is a particular case of the MULTIBR ANCH tree, where $\mathrm{n}_{\sigma}$ is constant. The total number of scenarios $\mathrm{S}$ of the problem is therefore:

$$
S=\prod_{\sigma=1}^{S T-1} n_{\sigma}
$$

- binary

A binary tree is such that at each time stage, two possible outcomes of the random data can be observed. The total number of scenarios $\mathrm{S}$ of the problem has to satisfy the following equality:

$$
S=2^{S T-1}
$$

- twostage $\left\{n_{s}\right\}_{\text {opt }}$

If the model is a two-stage recourse model, then the tree structure can be declared using the twostage keyword. This is equivalent to define an nway $\{n s\}$ tree, where $n s$ is the number of scenarios for the model.

\section{Random parameters}

The random parameters of the model have to be identified and distinguished from the deterministic model data. If the model is scenario based, every parameter of the model which is random, needs to be explicitly identified as such. Also, the scenario dimension must be added to the indexing list of the parameter.

random param name indexing attributesopt;

In our deterministic example of section 3.1, the demand parameter is defined as:

param d $\{$ Prod, Deal, Time, Scen $\} \quad>=0 ; \quad$ \#demand

Using the random keyword, we explicitly redefine the parameter as a random variable:

scenarioset $\mathrm{Sc}:=1 . \mathrm{NS}$;

random param d $\{$ Prod, Deal, Time, Scen $\}>=0 ; \quad$ \#demand

The particular nature of the multistage stochastic programs with recourse introduces the choice between diverse forms of presenting the data values for the random parameters. These forms can be either compact or expanded. The compact form assumes that the scenario data can present itself in the form of a 2-dimensional matrix (tree matrix) where only some of the entries exist. The matrix forms 
a grid where the columns represent time periods and the rows represent scenarios. The conditions under which such a matrix, partially entered, can represent a tree may be stated as follows:

1. Entry $(1,1)$ must exist

2. Entries $(T, s)$ exist for all scenarios $s=1 . . S$, where $T$ is the number of time periods and $\mathrm{S}$ the number of scenarios.

3. If entry $(t, s)$ exists for some $t<T$, then also does entry $(t+1, s)$.

Each entry $(t, s)$ of the matrix represents the realisation of the random parameter at time period $t$ under scenario s. An entry can consist of a single value or a vector of values if the random parameter is indexed over sets other than the time set and the scenario set.

For instance, let us consider a 3 time period horizon and random parameter $p$, which takes the (known) value 10 in the first time period. Let us consider that 2 realisation of the random parameter can be observed at each time period $t=2 . .4$, as in figure Figure 12:

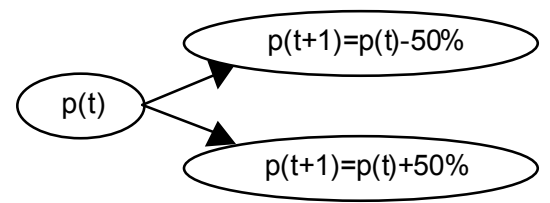

Figure 12. Simple event tree rule.

This rule defines a simple scenario generator, whereby a binary tree can be constructed, as in figure Figure 13.

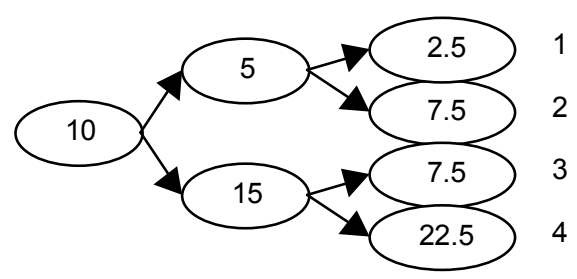

Figure 13. Binary scenario tree.

The (sparse) matrix representing the data for this scenario tree is shown in Table 4:

\begin{tabular}{|c|c|c|c|}
\hline $\mathrm{t}=1$ & $\mathrm{t}=2$ & $\mathrm{t}=3$ & \\
\hline 10 & 5 & 2.5 & $\mathrm{~s}=1$ \\
\hline & & 7.5 & $\mathrm{~s}=2$ \\
\hline
\end{tabular}




\begin{tabular}{|c|c|c|c|}
\hline & 15 & 7.5 & $\mathrm{~s}=3$ \\
\hline & & 22.5 & $\mathrm{~s}=4$ \\
\hline
\end{tabular}

Table 4.Compact scenario data.

Such matrix can be represented in SAMPL in a row-wise fashion as:

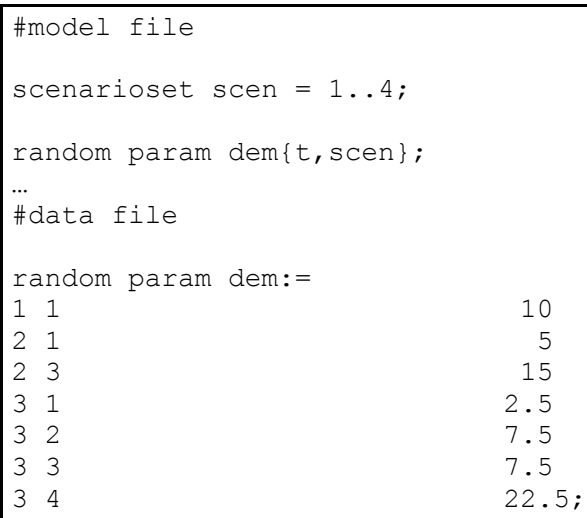

Although the data format here illustrated avoids any possible redundancy, it often happens that the scenario data are provided in what we call expanded form. This can be thought as the tree matrix above, where all entries are given. The expanded matrix relative to the example previously investigated is shown in Table 5:

\begin{tabular}{|c|c|c|c|}
\hline $\mathrm{t}=1$ & $\mathrm{t}=2$ & $\mathrm{t}=3$ & \\
\hline 10 & 5 & 2.5 & $\mathrm{~s}=1$ \\
\hline 10 & 5 & 7.5 & $\mathrm{~s}=2$ \\
\hline 10 & 15 & 7.5 & $\mathrm{~s}=3$ \\
\hline 10 & 15 & 22.5 & $\mathrm{~s}=4$ \\
\hline
\end{tabular}

Table 5. Expanded scenario data.

The data for the random parameter $\operatorname{dem}\{\mathrm{T}, \mathrm{S}\}$ are expressed in tabular form as follows:

\begin{tabular}{|lrrr|}
\hline \#data file & & & \\
random param dem (tr) $:=$ & & & \\
& 1 & 2 & 3 \\
1 & 10 & 5 & 2.5 \\
2 & 10 & 5 & 7.5 \\
3 & 10 & 15 & 7.5 \\
4 & 10 & 15 & 22.5 \\
\hline
\end{tabular}

\section{Chance constraints}

Probabilistic (or chance) constraints are characterised by randomness in some of the coefficients and by a level $\beta$ which indicates the probability of satisfying the constraint. Chance constraints are defined using the following syntax:

chance indexingopt constraint_name relop expression; 
where relop is one of $\leq, \geq$. Consider the following deterministic constraint vector definition in AMPL:

satisfy demand $\{j$ in Prod, $\mathrm{k}$ in Deal, $t$ in Timem, $\mathrm{s}$ in Scen\}: sum $\{i$ in $\mathrm{Fact}\} \mathrm{z}[j, \mathrm{i}, \mathrm{k}, \mathrm{t}, \mathrm{s}]$ $>=d[j, k, t, s]$

Assuming that $d[j, k, t, s]$ is a random parameter with realisations given in the form of scenarios, the constraint can be transformed into a probabilistic one as in the following example:

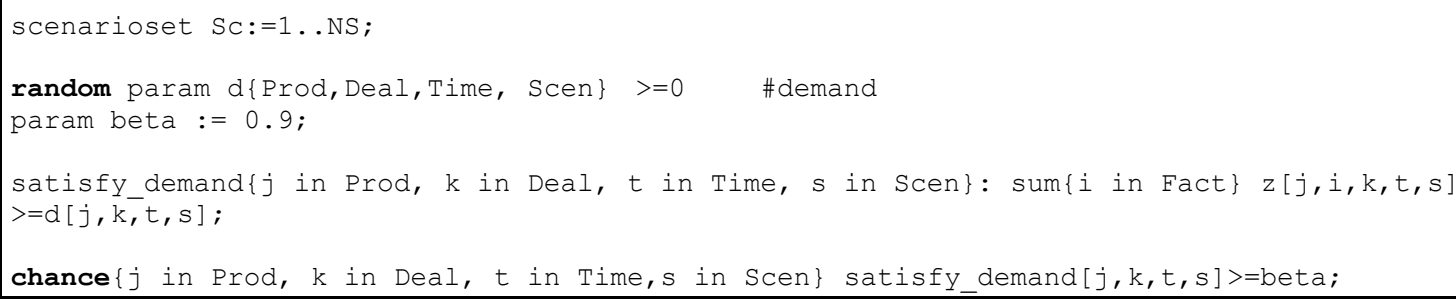

The chance statement indicates that each row of the constraint vector satisfy_demand has to be satisfied with probability greater than 0.9 .

\subsection{Implementation of the illustrative examples in SAMPL}

In this section, we combine the AMPL standard constructs with the proposed SAMPL extension in order to formulate the illustrative example of section 3 in its two-stage, multistage and chance constrained versions. More specifically, we first formulate the underlying deterministic model (Wait and See) in AMPL, and then add the stochastic information using the SAMPL constructs.

\section{The underlying deterministic model}

The underlying deterministic model associated with the problem illustrated in section 3 can be formulated in AMPL as follows:

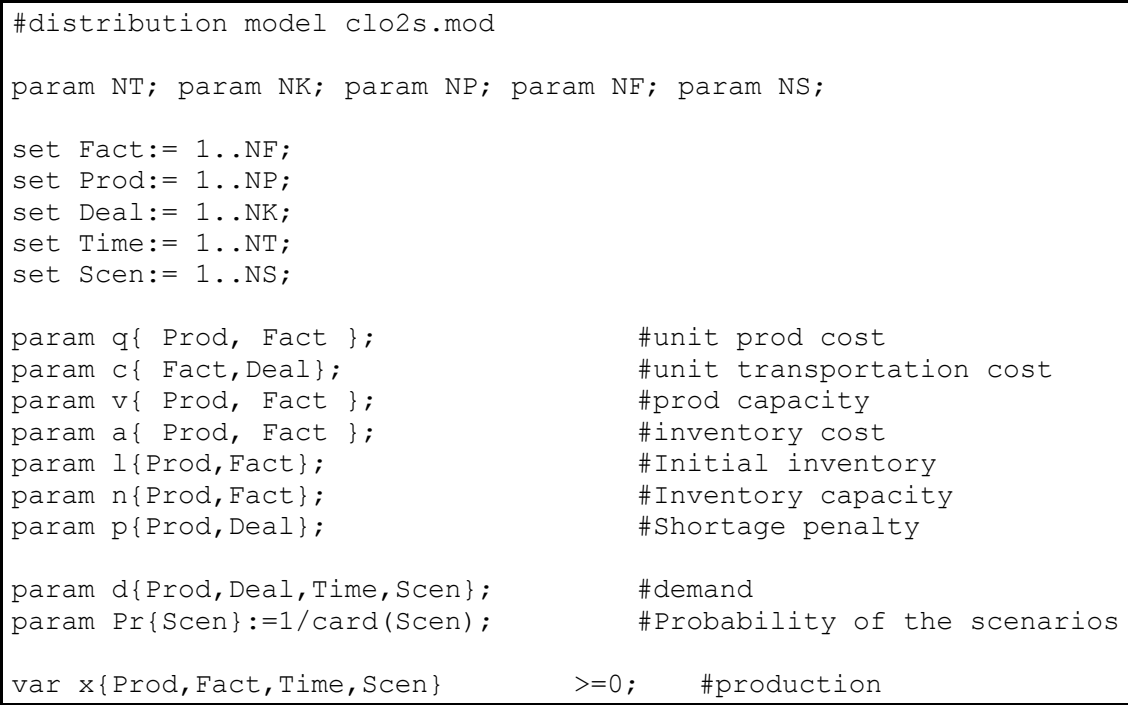




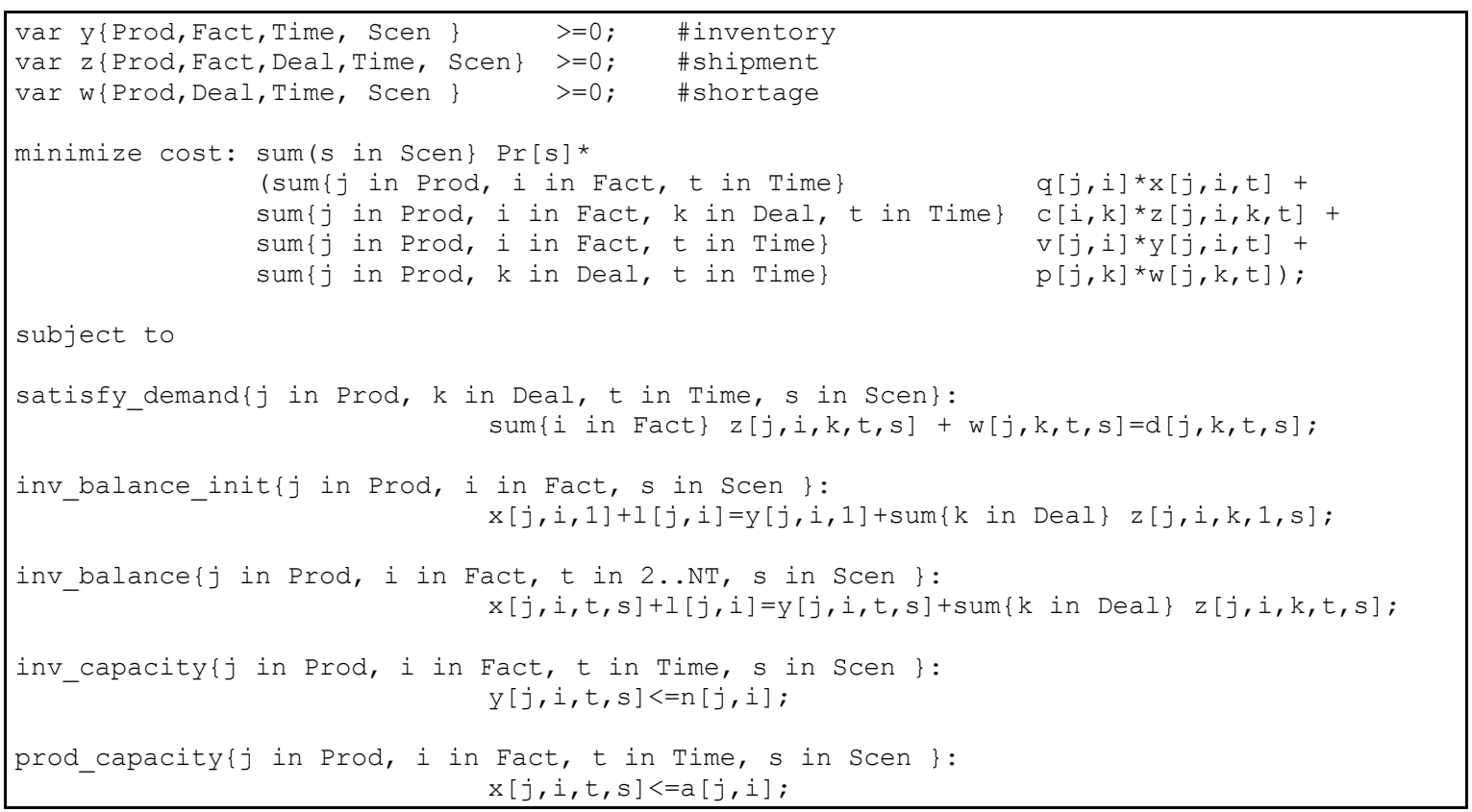

\section{The two stage recourse model}

To illustrate the use of the new constructs for stochastic programming described in the previous section, we modify the above deterministic model as follows:

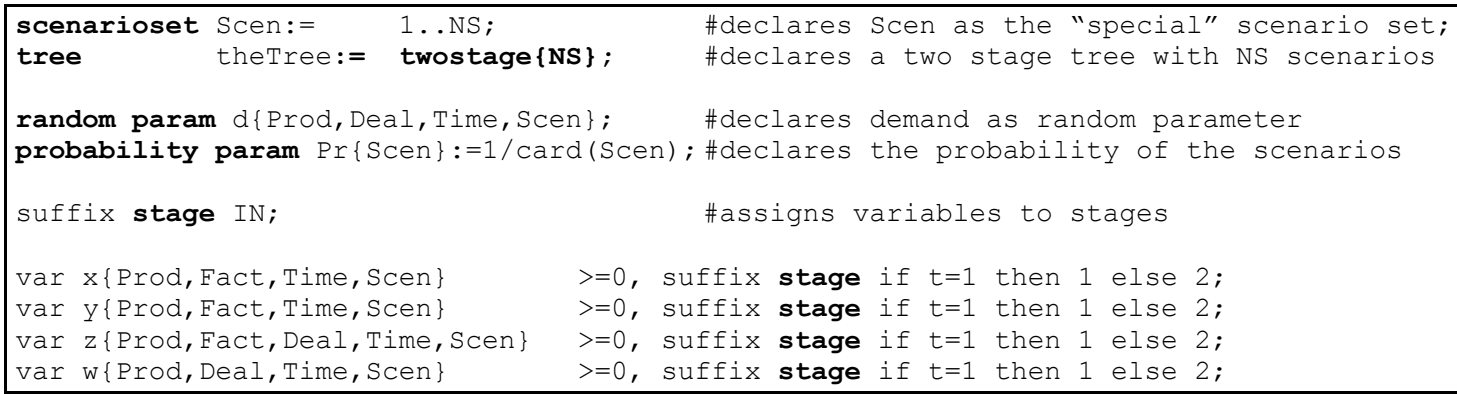

The set Scen is defined using the scenarioset keyword, which identifies it as a special set. The tree keyword is used to define the structure of the scenario tree as a two-stage (fan) tree.

The parameter $\mathrm{d}$ is declared using the random param keyword, while the probability vector $\operatorname{Pr}$ is redefined as probability param. Finally, the variables are partitioned into different stages by adding of the suffix stage to their definition.

\section{From two stage to multi stage}

Transforming the two-stage model to a multistage formulation for the same problem is very easily achieved using the SAMPL constructs. It is necessary to disaggregate the stages, and assign variables of different time periods to distinct stages. The tree structure of the model is also revised to that of the multistage tree of Figure 6. The resulting stochastic framework is set out as follows: 


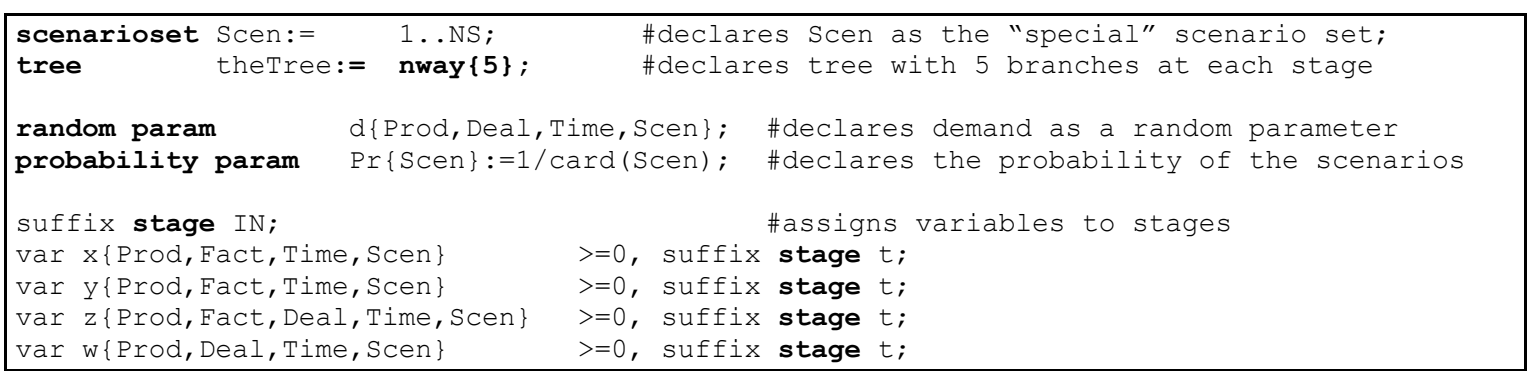

\section{Chance Constrained Problem in SAMPL}

The chance-constrained version of the same problem is also formulated using the extended language constructs. The underlying deterministic model is first revised in the following way:

1. Change the satisfy_demand constraint to allow for shortages. (This means that variable $w$ is not required any longer, and that the shortage cost is also removed from the objective function)

2. Define a reliability parameter which represents $\beta$ of equation (17).

A few additional SAMPL statements are added, including the declaration of the constraints named satisfy_demand as chance in the stochastic framework. The resulting model is set out below.

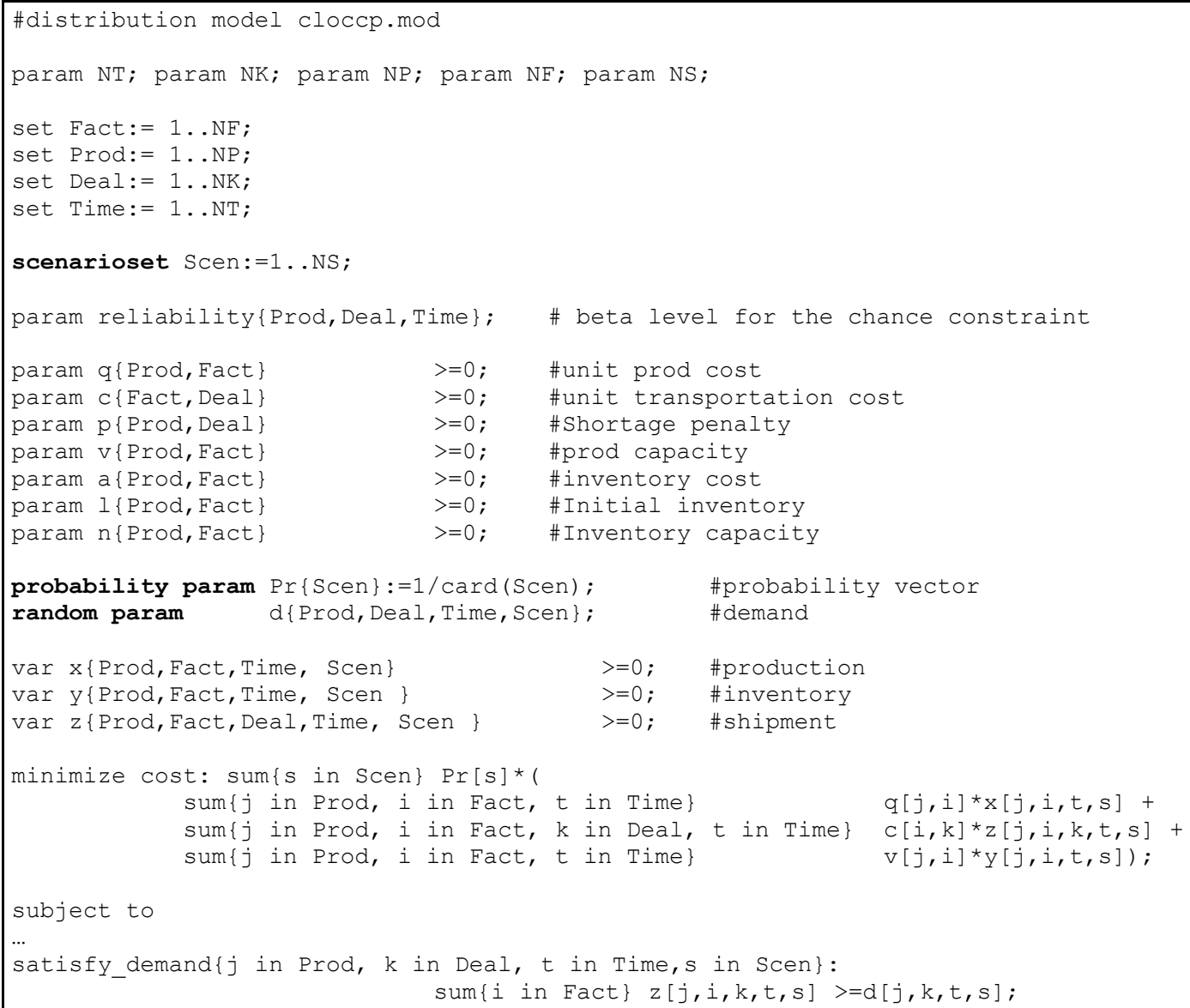




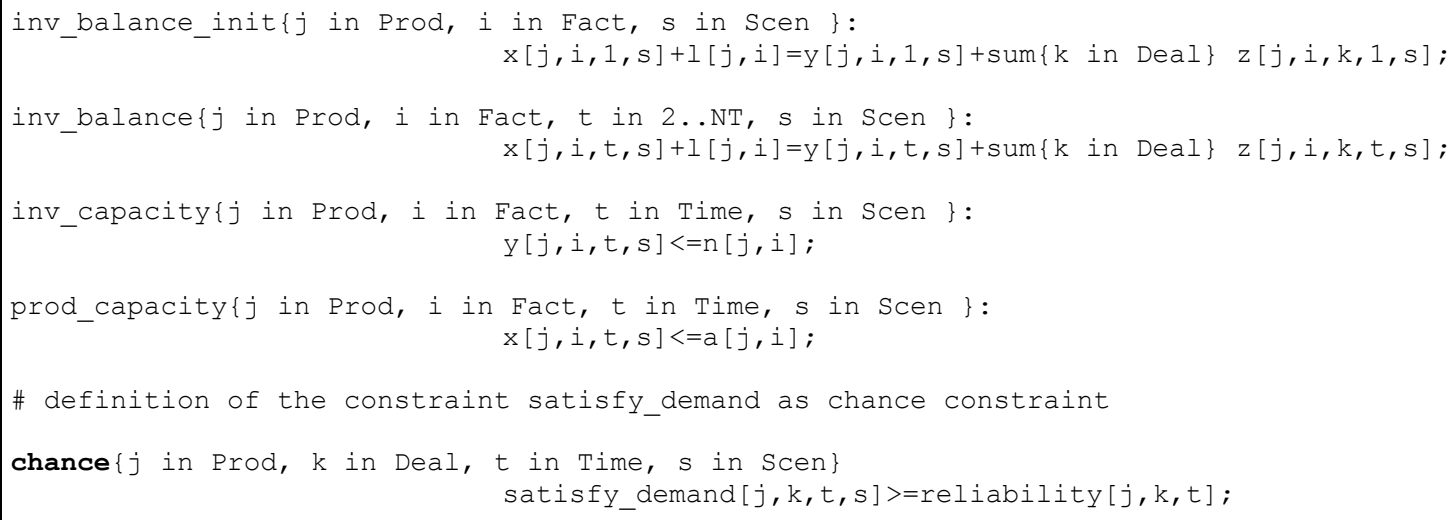

\section{Implementation of the language extensions}

Algebraic modelling languages are supported by modelling systems, which provide connectivity to data sources and solvers. The developers of algebraic modelling languages do not seem to be yet ready to undertake the laborious effort needed to extend their systems to include stochastic programming constructs. Some key aspects need to be taken into account:

- The inadequacies of existing matrix representation formats for SP problems, used to connect solvers to the modelling systems.

- The problem of linking modelling systems with scenario generators.

- The risk of introducing languages features which are not yet fully accepted by the OR community, nor by the developers themselves.

In this section, we address the first two issues and briefly introduce our SPInE system, which implements the language extensions presented in this paper and is readily connected with SP solvers and scenario generators.

\subsection{Interfacing modelling systems and solvers}

The main problem associated with the interfacing of modelling system and specialised SP solvers is the representation and communication of the SP model instances.

\section{Representation of SP model instances.}

The SMPS format as introduced in (Birge, Dempster et al. 1988) enables the compact representation of large scale stochastic programming problems, including scenario based recourse problems. However, in (Gassmann and Schweitzer 2001) the authors illustrate a number of shortcomings associated with this standard. The authors propose an extended SMPS format with added 
representational power which overcomes most of the problems associated with the original standard. In this research, however, other types of problems have been identified, which cannot be directly represented using the SMPS standard, nor its extended version.

\section{Recourse problems without first stage constraints.}

Consider the following simple problem, which is a variant of the newsboy problem:

The newsboy needs to decide, every day, what is the optimal number of newspaper $x$ to buy in order to maximize the profits. The demand $D$ is not known with certainty. A set of scenarios $S$ for the demand is given. The outcomes of the demand for each scenario $s$ in $S$ are defined as $D_{s}$ and the associated probability is $P_{s}$. Each copy of the newspaper is characterized by a unit selling price $R$ and a unit purchase cost $C$. Unsold copies $y_{s}{ }^{+}$represent a loss for the newsboy. The eventual shortfall of copies is indicated with $y_{s}$ and is characterised by a penalty term in the objective function. Shortfall and excess are only revealed once the demand is known, hence $x$ is a first stage variable, while $y_{s}{ }^{+}$and $y_{s}$ are second stage variables. The algebraic formulation of the two-stage model can be stated as follows:

$$
\max \text { profit }=(R-C) x-(R-C) \sum_{s \in S} P_{s} y_{s}^{-}-R \sum_{s \in S} P_{s} y_{s}^{+}
$$

s.t.

$x+y_{s}^{-}-y_{s}^{+}=D_{s} \quad \forall \mathrm{s} \in \mathrm{S}$

where the objective is to maximise the profit, expressed as the profit of selling all the newspaper purchased, minus the expected value of the (false) profit generated by the unsold copies and the expected value of the shortfall penalty. The ordered matrix of this problem, for $|S|=3$ is the following:

\begin{tabular}{|c|c|c|c|c|c|c|c|}
\hline$x$ & $y_{1}^{-}$ & $y_{1}^{+}$ & $y_{2}^{-}$ & $y_{2}^{+}$ & $y_{3}^{-}$ & $y_{3}^{+}$ & \\
\hline R-C & $\mathrm{P}_{1}(\mathrm{R}-\mathrm{C})$ & $\mathrm{P}_{1} \mathrm{R}$ & $\mathrm{P}_{2}(\mathrm{R}-\mathrm{C})$ & $\mathrm{P}_{2} \mathrm{R}$ & $\mathrm{P}_{3}(\mathrm{R}-\mathrm{C})$ & $\mathrm{P}_{3} \mathrm{R}$ & \\
\hline 1 & 1 & -1 & & & & & $\mathrm{D}_{1}$ \\
\hline 1 & & & 1 & -1 & & & $\overline{\mathrm{D}_{2}}$ \\
\hline 1 & & & & & 1 & -1 & $\mathrm{D}_{3}$ \\
\hline
\end{tabular}

This degenerate type of models is characterised by two difficulties, namely the fact that the first stage constraints are missing and that the first stage problem is unbounded. The first feature precludes the possibility of representing the model using the SMPS format. Indeed, the time file of the SMPS requires the specification of the top-left element of the block of non-zeroes identified by the 
variables and constraints which belong to each stage. In this case, the block relating to the first stage is empty, hence SMPS cannot be adopted.

Also, if the first stage problem is unbounded, solution algorithms based on Benders' decomposition may fail. In general, these algorithms try to solve a master problem associated with the first stage in order to obtain a feasible first stage solution, which is then refined by solving sequences of subproblems (associated with second stage) which are parametric in the first stage decision. The solution of the subproblems leads to cuts which are added to the master problem until the optimum solution is found. If the master is unbounded, the algorithm may fail even if the problem as a whole is feasible. An approach to deal with this problem is to create some artificial bounds on the first stage variables during the very first iteration of the decomposition algorithm. This may provide a feasible starting decision for the subsequent solution of the subproblems.

\section{Global constraints}

The term "global constraint" is used in the context of this research to identify a class of constraints which may be required in some scenario based stochastic programming problems. A global constraint is a constraint which involves decision variables associated with different nodes of the scenario tree. Consider for instance the following two-stage recourse problem:

$$
\min c x+\sum_{s \in S} p_{s} f_{s} y_{s}
$$

s.t.

$a x=b$

$u_{s} x+t_{s} y_{s}=h_{s} \quad \forall \mathrm{s} \in \mathrm{S}$

$\sum_{s \in S} p_{s} r_{s} y_{s} \leq k$

The associated matrix for $|S|=3$ is the following:

\begin{tabular}{|l|l|l|l|l|}
\hline \multicolumn{1}{|c|}{$x$} & \multicolumn{1}{|c|}{$y_{1}$} & \multicolumn{1}{c|}{$y_{2}$} & \multicolumn{1}{c|}{$y_{3}$} & \multirow{2}{*}{} \\
\hline$c$ & $p_{1} f_{1}$ & $p_{2} f_{2}$ & $p_{3} f_{3}$ & \\
\hline$a$ & & & & $b$ \\
\hline$u_{1}$ & $t_{1}$ & & & $b_{1}$ \\
\hline$u_{2}$ & & $t_{2}$ & & $h_{2}$ \\
\hline$u_{3}$ & & & $t_{3}$ & $h_{3}$ \\
\hline & $p_{1} r_{1}$ & $p_{2} r_{2}$ & $p_{3} r_{3}$ & $k$ \\
\hline
\end{tabular}


The last row links variables belonging to three different scenarios. One of the underlying assumptions of SMPS is that the blocks of the matrix which relate to different scenarios need to be completely separable, therefore these types of problems cannot be represented using the SMPS format. Global constraints are generally used to introduce restrictions on the expected value of some function of the decision variables. Typical examples of such functions are risk measures such as the Expected Downside Risk or the Conditional V alue at Risk (CVaR).

A modelling system which supports stochastic programming modelling should therefore take into account the issues related to the representation of the model instances. In the specific case of global constraints, the modelling system should be able to detect such expressions and move the constraint into the objective function with as a penalty term.

\section{New directions: XML}

The limitations of the SMPS format in terms of representational power have become apparent over the years, and some extensions have been proposed (Gassmann and Schweitzer 2001). Recently, researchers have expressed significant requirements for an enhanced standard, specifically for the purpose of communicating mathematical programs. A new approach, which takes into consideration contemporary technical assumptions, is based on eXtended Mark-up Language (XML). The wealth of functionality and software available for exploiting XML is an important factor in the acceptance of an XML based mathematical programming standard. There are currently at least three XML vocabularies proposed for this role: FML (Fourer, Lopes et al. 2003), OptML (Kristjansson 2001) and SNOML (Lopez and Fourer 2001). Both support linear and non-linear optimisation models, but in addition, SNOML is also able to capture stochastic programming models with recourse, chanceconstrained models, and also constraint logic programming models.

At this stage, OptML and SNOML are not fully designed nor accepted by the research community; however, they seem to be the most promising approaches towards a new standard for the representation and communication of mathematical programming problems, including stochastic programming models.

\subsection{Communicating with scenario generators}

\section{Linking scenario generators with modelling systems}

The creation of the scenario tree used in multistage stochastic programming is usually carried out by specialised applications called scenario generators. While the algebraic optimisation model captures the logic of the application's domain, the scenario generators are special purpose applications developed to capture the randomness properties of the application's domain. Typically, a consumer product 
supply chain model and an energy distribution model both require scenarios of forecast demand, but the factors which influence the demands and the forecasting models may be very different. Again in finance applications the asset prices under consideration may be generated using different models of credit risk (Jobst 2002), interest rate risk or other considerations. In designing an SP modelling system, one goal is therefore to develop an appropriate parameter passing interface which enables the connection of diverse special purpose scenario generators (which capture valuable domain knowledge) to the modelling system

A scenario generator $\varphi$ captures in a procedural form a domain-specific model of randomness. In particular it uses historical information, an event tree structure and some other specification parameters to forecast a set of scenarios. The main groups of parameters can thus be separated as:

H : History,

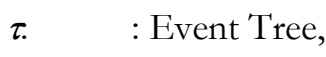

$\boldsymbol{\theta} \quad$ : Remaining Parameters.

The set of scenarios $\Xi$ is then seen as the collection of scenarios which are output by the generation procedure:

$$
\varphi(H, \tau, \theta) \Rightarrow \Xi
$$

The data associated with the set of scenarios $\Xi$ are implicitly structured as the event tree $\tau$. This event tree is usually specified in terms of a time horizon $\mathrm{T}$ and the number of outcomes of the random parameters per time period. Trees with arbitrary structures are typically defined by specifying the underlying node structure. The historical data $H$ are often used to determine an underlying probability distribution for the random parameters. Historical data may also contain the initial values (that is, the values assumed by the random parameters in the root node of the scenario tree). If the historical data are not used to determine the probability distributions, the set of remaining parameters $\theta$ may include the type of distribution and estimates of its moments.

The tree structured outcomes of the random parameters $\tau$, induce a similar structure in the underlying optimum decision making problem, as the variables and constraints are dependent on the realisations of the random parameters. This is a central question which needs to be addressed when modelling stochastic programming problems using algebraic modelling languages: the algebraic form of the SP model requires the specification of the 'variable and constraint' tree structure, labelled as $\tau$ '. An extended AML should provide constructs for the specification of $\tau$ in the SP model. For consistency, the two trees $\tau$ and $\tau^{\prime}$ need to be congruent. In other words, it has to be ensured that the 
event tree structure $\tau$ used by the special purpose scenario generator is 'compatible' with the $\tau$ ' specified in the SP model and vice versa (Valente, Mitra et al. 2001). The requirement for scenario generator parameter passing and tree consistency conditions are illustrated in Figure 14. When a special purpose scenario generator is connected to the modelling system, the two trees $\tau$ and $\tau$ are compared for consistency. The scenario generator then creates the set of scenarios and the associated probabilities $p(\omega)$.

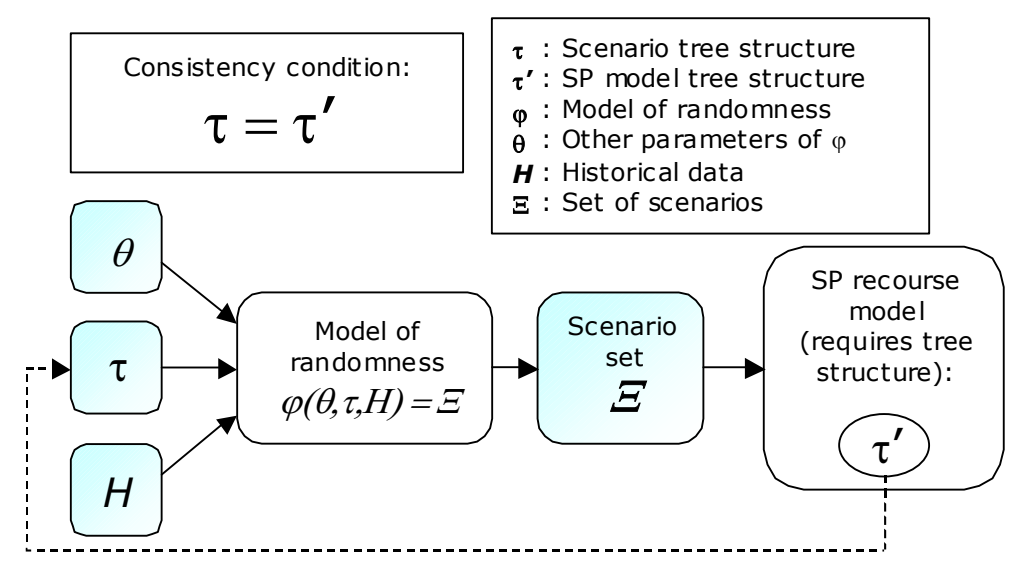

\section{Figure 14. Scenario Generation and Tree Consistency}

If the scenario generation procedure is kept separated from the modelling system, the consistency of $\tau$ and $\tau^{\prime}$ can be ensured if the algebraic specification of the model enables the definition of $\tau$ as a special model parameter. This is then instantiated using $\tau$, which therefore needs to be explicitly provided by the scenario generator along with the scenario data $\Xi$. The modelling system should be able to use the information in the special parameter $\tau^{\prime}$ to introduce the structure in the model's variables and constraints, either via explicit non-anticipativity constraints, or implicitly by creating the appropriate variables and constraints for each node of the tree. This approach is consistent with the modelling process using systems based on declarative algebraic languages: the algebraic model and the data (deterministic data, scenarios data and the tree structure, in this case) are kept separated.

A desirable aspect of modern algebraic modelling languages is to include procedural features; for instance, many procedural constructs enable closer coupling with the solvers. The same concept could be applied to enable a closer coupling of modelling system with external scenario generators. The tree structure $\tau$, the historical data $\mathrm{H}$ and the remaining parameters $\theta$ are defined using the algebraic modelling language, and the modelling system provides procedural constructs to "call" the scenario generator passing the parameters. The resulting scenario data is then consistent with $\tau^{\prime}$ and can be used to instantiate the stochastic programming model. Unfortunately, these features are not directly available in modern modelling systems. A major focus of this research is to introduce special 
purpose language constructs for the definition of scenario based recourse problems and an integrated system which supports these language constructs. These together enable the seamless integration of algebraic modelling with scenario generation procedure using the paradigms discussed in this section.

\subsection{Integrated environments: SPInE}

Our implementation of the SAMPL language processor is the core of the modelling subsystem of our Stochastic Programming Integrated Environment (SPInE) (Valente, Mitra et al. 2002).

SPInE is divided into four main subsystems, namely Scenario Generation, Modelling, Solver, Results Analysis and the overarching Control module.

\section{Scenario Generation}

SPInE is designed to interface with scenario generators which supply the scenario data in ODBC databases or text files. An important aspect of the scenario generation interface is to establish the consistency between the SP model tree $\tau^{\prime}$ and the data path tree $\tau$ underlying the scenario generation (see Section 5.2).

\section{Modelling subsystem}

The modelling subsystem is designed to support the SAMPL language extensions introduced in this report. Similarly, it supports the SMPL stochastic extensions of the MPL language. A software module called Stochastic Program Generator (SPG) combines two separate parsers and a matrix generator. SPG processes together the algebraic models and the scenario data set to create an instance of the model in either SMPS format or in the Stochastic Intermediate Representation (SIR). The SPG module makes use of an underlying modelling engine, specifically OptiMax for the support of SMPL models and a comparable AMPL-based COM object, developed by our research group, for models prepared in SAMPL. The modelling system interacts directly with the scenario generators for the stochastic data can connect to database systems or data files which store the deterministic data relating to the core model of the SP problem.

\section{Solver subsystem}

Given a Stochastic Programming problem with recourse, formulated in SAMPL, the FortSP stochastic solver embedded in SPInE (Poojari, Ellison et al. 2002) provides the solution to three related classes of models:

- Here and Now

- Scenario Analysis (Wait and See) 
- $\quad$ Expected Value

For each of these, there is more than one possible solution algorithm. All underlying LPs may be solved using the Sparse Simplex algorithm (SSX) or the Interior Point Method (IPM). The Here and Now problem may be solved using Benders Decomposition, Lagrangean Relaxation or via the Deterministic Equivalent problem. The solver is also able to report the stochastic measures EVPI and VSS.

\section{Results analysis}

A critical phase in the development of stochastic models is the analysis of the solutions. The integration with database systems enables the exploitation of the Data Manipulation Languages (DML) which usually accompany the DBMS for the development of customised viewers and advanced data analysis tools. The SP Reporter (SPR) module of SPInE allows the user to export solution vectors using standard ODBC or using text files. The volume of the solution results produced by the stochastic solver can be very large. In fact, each decision variable has an associated optimal activity and reduced cost, for each stage and for each scenario. The investigator might be interested only in a subset of the solutions (e.g. the first stage strategic decisions). SPR provides filtering functionality which is used to transfer only the relevant decision data to the DBMS.

\section{Control Module and Graphical User Interface}

Each module in SPInE can be run as an independent application through script files. A control module including a Graphical User Interface (GUI) has been developed and can be used to investigate SP problems. The main subsystems of SPInE, namely the SP instances generator SPG, the stochastic solver FortSP and the solution Reporter SPR have also been wrapped in a dynamic link library, which enables the rapid development of embedded applications.

\section{Conclusions and directions for future research}

Modelling and solving stochastic programming problems is a challenging task. Stochastic programming models can be transformed into deterministic equivalent formulations by discretising the support of underlying continuous probability distributions. However, it is often impossible to take advantage of the existing modelling tools and LP/MIP solvers to formulate and process these models. In this report, we have identified a number of modelling requirements for stochastic programming. Taking these into account, we have designed an approach to extending algebraic modelling languages for the formulation of SP models in a natural and concise way and we have shown how this approach can be applied to the AMPL modelling language with a practical example. 
The difficulties associated with the modelling of the random parameters for SP problems are discussed, and some insight is gained into the relations between scenario trees and SP model structures; thereby the requirements for the seamless integration of scenario generators and modelling systems are identified. Current algebraic modelling languages are required to capture the scenario structures of SP models. The problem of representing instances of stochastic programming models in formats which can be exploited by specialised solution algorithms are also studied.

A number of issues which remain to be addressed have been identified in respect of the process of modelling, solution and analysis of stochastic programming problems.

The language constructs proposed in this research only address the class of scenario-based stochastic programming problems, while distribution-based problems also need to be considered. The definition of the random parameters using probability distributions needs to take into account the complexity introduced, for instance, in multistage problems. In these models, there might be dependency of the random variables across stages, which implies the use of conditional probabilities. The integration of scenario generators requires further investigation. This aspect is strictly connected with the possibility of defining models of randomness directly in the modelling language. An alternative would be the introduction of a mechanism (eventually based on new language constructs) for the control of the scenario generation in algebraic modelling languages which support procedural statements. A library of standard scenario generators which readily connects to the SPInE system is under way. This will enable practitioners to truly deploy the system for a complete investigation of stochastic programming problems, and will also expand the capabilities of the simulation framework. 


\section{References}

Birge, J. R., M. A. H. Dempster, H. I. Gassmann, E. Gunn, A. J. King and S. W. Wallace (1988). "A standard input format for multiperiod stochastic linear programs." Mathematical Programming Society Committee on Algorithms Newsletter 17, 1-19.

Bisschop, J. J. and M. Roelofs (1999). "AIMMS: The Language Reference." Paragon Decision Technology BV, Haarlem, The Netherlands.

Bosetti, V., E. Messina and P. Valente (2002). "Optimisation technologies and environmental applications." submitted to the IMA Journal of Management Mathematics.

Brooke, A., D. A. Kendrick, A. Meeraus and R. Raman (1998). "GAMS : a user's guide,Release 2.50."

Buchanan, C. S., K. I. M. McKinnon and G. K. Skondras (2001). "The Recursive Definition of Stochastic Linear Programming Problems within an Algebraic Modeling Language." Annals of Operations Research 104(1), 15-32.

Charnes, A. and W. W. Cooper (1959). "Chance-constrained programming." Management Science 5, 73-79.

Entriken, R. (2001). "Language Constructs for Modeling Stochastic Linear Programs." Annals of Operations Research 104(1), 49-66.

Eppen, G. D., R. K. Martin and L. Schrage (1989). "A Scenario Approach to Capacity Planning." Operations Research 37(4), 517-527.

Escudero, L. F., E. Galindo, G. Garcia, E. Gomez and V. Sabau (1999). "Schumann, a modeling framework for supply chain management under uncertainty." European Journal of Operational Research 119(1), 14-34.

Fourer, R. (1996). "Proposed new AMPL features - stochastic programming extensions." www.ampl.com/cm/cs/what/ampl/NEW/FUTURE/stoch.html.

Fourer, R. (2001). "Model-level directives for generating alternative formulations of stochastic programs." IX International Conference on Stochastic Programming, Berlin, Germany.

Fourer, R., D. M. Gay and B. W. Kernighan (1993). "AMPL : a modeling language for mathematical programming." Danvers Mass Boyd Fraser Pub. CO,.

Fourer, R., L. Lopes and K. Martin (2003). "A W3C XML schema for linear programming." Northwestern University and The University of Chicago, (work in progress).

Gassmann, H. I. and A. M. Ireland (1995). "Scenario formulation in an algebraic modelling language." Annals of Operations Research 59, 45-76.

Gassmann, H. I. and A. M. Ireland (1996). "On the formulation of stochastic linear programs using algebraic modelling languages." Annals of Operations Research 64, 83-112.

Gassmann, H. I. and E. Schweitzer (2001). "A comprehensive input format for stochastic linear programs." Annals of Operations Research 104(1), 89-125. 
Gay, D. M. (2001). "Random parameters - a step toward conveniently expressing some stochastic programming problems." IX International Conference on Stochastic Programming, Berlin, Germany.

Jobst, N. (2002). On credit risk modelling, simulation, optimisation. $\mathrm{PhD}$ thesis, Department of Mathematical Sciences. Uxbridge, UK, Brunel University.

King, A. J. (1994). "SP/OSL Version 1.0 Stochastic Programming Interface Library: User' s Guide, Research Report RC 19757 (87525) 9/26/94 Mathematics." T.J Watson Research Center, Yorktown Heights, NY.

Kristjansson (2001). "How New Technologies such as XML and SOAP allow OR to provide Webbased Services." INFORMS Roundtable Savannah, Georgia.

Lopes, L. and R. Fourer (2003). "A modeling language for stochastic programming." ISMP 2003, Copenhagen, Denmark.

Lopez, L. and R. Fourer (2001). "An XML-Based Format for Sharing Mathematical Programs." INFORMS international conference on Miami, November 2001.

Lucas, C., E. Messina and G. Mitra (1997). "Risk and Return Analysis of a Multi-period Strategic Planning Problem." Lecture notes in economics and mathematical systems (445), 81.

Maximal Software (2002). "MPL Modelling System, Release 4.2." USA.

Messina, E. and G. Mitra (1997). "Modelling and analysis of multistage stochastic programming problems: A software environment." European Journal of Operational Research 101(2), 343-359.

Pereira, M. V. F. and L. M. V. G. Pinto (1985). "Stochastic Optimization of a Multireservoir Hydroelectric System: A Decomposition Approach." Water Resources Research 21(6), 779-792.

Poojari, C. A., E. F. D. Ellison and G. Mitra (2002). "A multi-stage stochastic programming solver." Working paper, CARISMA Centre for the Analysis of Risk and Optimisation Modelling Applications, Brunel University, London, UK.

Shapiro, J. (2001). "Modeling the Supply Chain." Duxbury Press, California.

Valente, P. (2002). Software tools for the investigation of stochastic programming problems. $\underline{\mathrm{PhD}}$ Thesis, Department of Mathematical Sciences. Uxbridge, UK, Brunel University.

Valente, P., G. Mitra and C. A. Poojari (2002). "A Stochastic Programming Integrated Environment (SPInE)." in S. W. Wallace and W. T. Ziemba (Eds): to appear in Applications of Stochastic Programming.

Valente, P., G. Mitra, C. A. Poojari and T. Kyriakis (2001). "Software Tools for Stochastic Programming: A Stochastic Programming Integrated Environment (SPInE)." TR 10/01, Department of Mathematical Sciences, Brunel University, London, UK.

Ziemba, W. T. and J. M. Mulvey, (Eds). (1998). "Worldwide asset and liability modeling." Cambridge University Press, Cambridge. 